(D)

Check for

updates

Cite as

Nano-Micro Lett.

(2020) 12:137

Received: 26 February 2020

Accepted: 1 June 2020

Published online: 30 June 2020

(C) The Author(s) 2020

\section{Soft Mesoporous Organosilica Nanoplatforms Improve Blood Circulation, Tumor Accumulation/ Penetration, and Photodynamic Efficacy}

\author{
Xin Peng ${ }^{1}$, Kun $\mathrm{Chen}^{3}$, Wanhua Liu ${ }^{1}{ }^{凶}$, Xiongfeng Cao $^{4}$, Mengru Wang ${ }^{1}$, Jun $\mathrm{Tao}^{3}$, \\ Ying Tian $^{2}$, Lei $\mathrm{Bao}^{5}$, Guangming $\mathrm{Lu}^{2}{ }^{凶}$, Zhaogang Teng ${ }^{2,3} \bowtie$ \\ Xin Peng and Kun Chen have contributed equally to this work. \\ $\bowtie$ Wanhua Liu, liuwanhua.com@126.com; Guangming Lu, cjr.luguangming@vip.163.com; Zhaogang Teng, \\ iamzgteng@njupt.edu.cn \\ 1 Jiangsu Key Laboratory of Molecular and Functional Imaging, Department of Radiology, Zhongda \\ Hospital, School of Medicine, Southeast University, Nanjing 210009, Jiangsu, People's Republic of China \\ 2 Department of Medical Imaging, Jinling Hospital, School of Medicine, Nanjing University, Nanjing 210002, \\ People's Republic of China \\ 3 Key Laboratory for Organic Electronics and Information Displays, Jiangsu Key Laboratory for Biosensors, \\ Institute of Advanced Materials, Jiangsu National Synergetic Innovation Centre for Advanced Materials, \\ Nanjing University of Posts and Telecommunications, Nanjing 210023, People's Republic of China \\ 4 Affiliated Hospital of Jiangsu University, Jiangsu University, Zhenjiang 212001, People's Republic of China \\ 5 Soft Matter and Interface Group, School of Engineering, RMIT University, Melbourne, VIC 3000, Australia
}

\title{
HIGHLIGHTS
}

- Soft mesoporous organosilica nanoplatforms modified with hyaluronic acid and cyanine 5.5 are constructed.

- Therapeutic efficacy of the soft nanoplatforms on tumor is higher both in vitro and in vivo after loading photosensitizer chlorin e6 compared to that of stiff counterparts.

ABSTRACT To date, the ability of nanoplatforms to achieve excellent therapeutic responses is hindered by short blood circulation and limited tumor accumulation/ penetration. Herein, a soft mesoporous organosilica nanoplatform modified with hyaluronic acid and cyanine 5.5 are prepared, denoted SMONs-HA-Cy5.5, and comparative studies between SMONs-HA-Cy5.5 (24.2 MPa) and stiff counterparts (79.2 MPa) are conducted. Results indicate that, apart from exhibiting a twofold increase in tumor cellular uptake, the soft nanoplatforms also display a remarkable pharmacokinetic advantage, resulting in considerably improved tumor accumulation. Moreover, SMONs-HA-Cy5.5 exhibits a significantly higher tumor penetration, achieving 30- $\mu \mathrm{m}$ deeper tissue permeability in multicellular spheroids relative

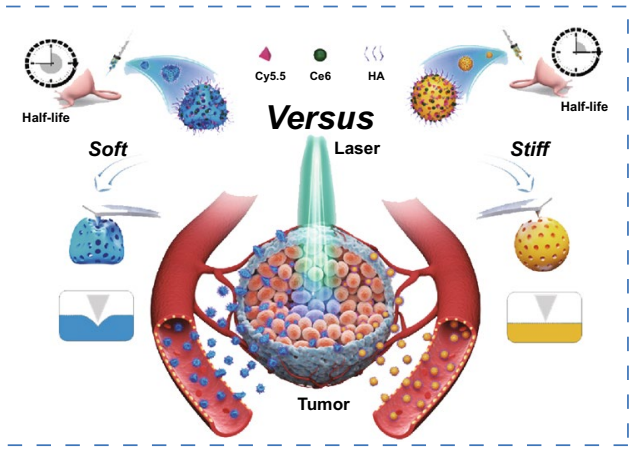
to the stiff counterparts. Results further reveal that the soft nanoplatforms have an easier extravasation from the tumor vessels, diffuse farther in the dense extracellular matrix, and reach deeper tumor tissues compared to the stiff ones. Specifically, the soft nanoplatforms generate a 16-fold improvement (43 vs. $2.72 \mu \mathrm{m}$ ) in diffusion distance in tumor parenchyma. Based on the significantly improved blood circulation and tumor accumulation/penetration, a soft therapeutic nanoplatform is constructed by loading photosensitizer chlorin e6 in SMONs-HA-Cy5.5. The resulting nanoplatform exhibits considerably higher therapeutic efficacy on tumors compared to the stiff ones.

KEYWORDS Mesoporous organosilica; Soft nanoplatform; Long circulation; Tumor accumulation; Tumor penetration 


\section{Introduction}

Cancer is a major disease that threatens human life. Recently, nanomedicine has enabled early diagnosis and precise treatment of various tumors, thereby bringing new hope for cancer management [1-4]. Generally, tumors possess an inherent pathologically leaky vasculature and defective lymphatics. Based on these characteristics, nanomaterial-based therapeutic agents that preferentially accumulate in the tumors, via the enhanced permeability and retention (EPR) effect, have been identified and are currently used for cancer treatment, with few side effects [5-8]. However, the nanomaterials systemically administered into the complicated biological systems need to go through multiple barriers, including circulating in the blood compartments, extravasating into perivascular tumor microenvironment and subsequently penetrating into the deep tumor tissues, until they were finally internalized by tumor cells [9-11]. During this complicated process, nanomaterials are likely to be sequestered and eliminated by the mononuclear phagocyte system (MPS) [12], hindered by dense tumor interstitial extracellular matrix (ECM) and the substantially elevated interstitial fluid pressure (IFP) [13, 14]. Consequently, there is a need to rationally design nanoplatforms that can overcome the aforementioned challenges and improve therapeutic outcomes. These platforms are required to meet the following requirements: (1) long blood circulation, (2) high tumor accumulation, (3) deep tumor penetration, (4) efficient internalization in tumor cells. A long blood circulation is one of the key factors for higher tumor accumulation [15, 16]. Meanwhile, deep tumor penetration and efficient cell internalization are essential for improving efficacy of therapeutic nanoplatforms [17-20]. To satisfy the above requirements, numerous platforms have focused on incorporating different physiochemical properties, including shape turning [21-24], size regulation [25-27] and surface modification [28-30] during fabrication of nanomaterials. However, varied approaches all had their own set of limitations, and cannot simultaneously address all issues. To date, short circulation and limited tumor penetration remain bottlenecks for nanoplatforms, thereby preventing effective therapeutic responses.

Mesoporous nanoparticles have become ideal candidates for constructing theranostic nanomaterials, owing to their tunable mesopore structures, sizable surface area and pore volume properties [31-33], that enable them effective vehicles for various molecular imaging probes and anticancer therapeutic agents [34-36]. Recently, research at our lab successfully obtained a soft hollow mesoporous organosilica nanoparticle [37]. Preliminary liquid cell transmission electron microscopy indicated that the nanoparticle undergoes morphological change, from spherical to oval, during cellular internalization, which generates a considerably higher cellular uptake. This advantage inspired us to construct soft nanoplatforms and systemically investigate their biological behaviors including blood circulation, tumor accumulation, tumor tissue penetration and the eventual therapeutic efficacy. To the best of our knowledge, this is the first study comprehensively exploring biological behaviors of soft mesoporous organosilica nanoplatforms.

In the work, a soft mesoporous organosilica nanoplatform, hereafter termed SMONs-HA-Cy5.5, has been prepared based on hyaluronic acid-(HA) and cyanine 5.5 (Cy5.5)modification. The outer HA layer comprises nanoplatforms with good dispersibility in aqueous solutions. The soft nanoplatforms have a lower Young's modulus (24.2 MPa), which is about $31 \%$ of the rigid counterparts MONs-HA-Cy5.5 (79.2 MPa). The soft structure grants the SMONs-HA-Cy5.5 double increased cellular uptake in MCF-7 cells and a longer mean residence time ( 9.09 vs. $3.94 \mathrm{~h}$ ) in the bloodstream. The significantly elongated blood circulation gives SMONsHA-Cy5.5 a twofold increase in tumor accumulation compared to MONs-HA-Cy5.5. More interestingly, SMONs-HACy5.5 exhibits $30 \mu \mathrm{m}$-deeper tumor penetration, than stiff counterparts in 3D tumor multicellular spheroids in vitro. Analysis of extravasation behavior reveals that SMONs-HACy5.5 have a 16-fold enhancement on diffusion distance in tumor interstitial extracellular matrix, relative to stiff ones that have lower perfusion rates and are thereby restricted in or near the tumor blood vessel. This indicates a significant boost of extravasation of SMONs-HA-Cy5.5 from the tumor blood vessel into the tumor microenvironment. Given the overall improved biological performance of the soft SMONs-HA-Cy5.5, we further load SMONs-HA-Cy5.5 with a photosensitizer chlorin e6, denoted SMONs-HA Ce6. Strikingly, the soft nanoplatform significantly improved the tumor therapeutic efficacy via photodynamic therapy following intravenous injection (Scheme 1). These results suggest that the soft mesoporous nanomaterials have the potential for developing nanotherapeutic platforms with more desirable biological performance and higher therapeutic efficiency. 


\section{Experimental Section}

\subsection{Materials}

Tetraethyl orthosilicate (TEOS), hexadecyltrimethyl ammonium bromide (CTAB, $25 \mathrm{wt} \%$ ), $\mathrm{NaOH}$, dioxane, and triphenylphosphine were bought from Sinopharm Chemical Reagent Co., Ltd. (Shanghai, China). Bis[3-(triethoxysilyl)propyl] tetrasulfide (TESPTS), Chlorin e6 (Ce6) and $N$-(3-dimethylaminopropyl)- $N^{\prime}$ ethylcarbodiimide hydrochloride (EDC), and 2',7'-dichlorofluorescin diacetate (DCFH-DA) were obtained from Sigma-Aldrich (St. Louis, MO, USA). N,N-dimethylformamide (DMF), concentrated ammonia aqueous solution (NH3H2O, 25-28 wt\%) and anhydrous ethanol were obtained from Nanjing Chemical Reagent Co., Ltd. (Nanjing, China). Concentrated hydrochloric acid ( $\mathrm{HCl}, 37$ wt\%) was brought from Shanghai Jiuyi Chemical Reagent Co., Ltd. (Shanghai, China). Cy5.5-maleimide was obtained from Seebio Biotechnology Co., Ltd. (Shanghai, China). $\mathrm{NH}_{2}$-maleimide was purchased from $\mathrm{Xi}$ ' an ruixi Biological Technology Co. Ltd. (China). $N$-hydroxysulfosuccinimide (NHS) was purchased from Shanghai Aladdin
Bio-Chem Technology Co., Ltd. (Shanghai, China). Hyaluronic acid (HA, MW 100-200 kDa) was purchased from Bloomage Freda Biopharm, Co., Ltd. (Shanghai, China). Singlet oxygen sensor green (SOSG) was purchased from Invitrogen (Carlsbad, CA, USA). Deionized water (Millipore) with a resistivity of $18 \mathrm{M} \Omega \mathrm{cm}$ was used for all experiments. Dulbecco's modified Eagle's medium (DMEM), phosphate-buffered solution (PBS), and cell counting kit-8 (CCK-8) were bought from Nanjing Keygen Biotech. Co., Ltd. (Nanjing, China). Heat-inactivated fetal bovine serum (FBS) was bought from Gibco Laboratories (NY, USA). MCF-7 cells were acquired from American Type Culture Collection (ATCC, Manassas, VA).

\subsection{Synthesis of Mesoporous Organosilica Nanospheres (MONs)}

MONs were synthesized according to a method reported in our previous work [37]. Typically, we prepared a solution composed of $25-28 \mathrm{wt} \%$ ammonia aqueous sol $(1.0 \mathrm{~mL})$, ethanol $(30 \mathrm{~mL})$, a concentrated CTAB $(0.16 \mathrm{~g})$, and water $(75 \mathrm{~mL})$. Next, mixed organosilica precursors comprising

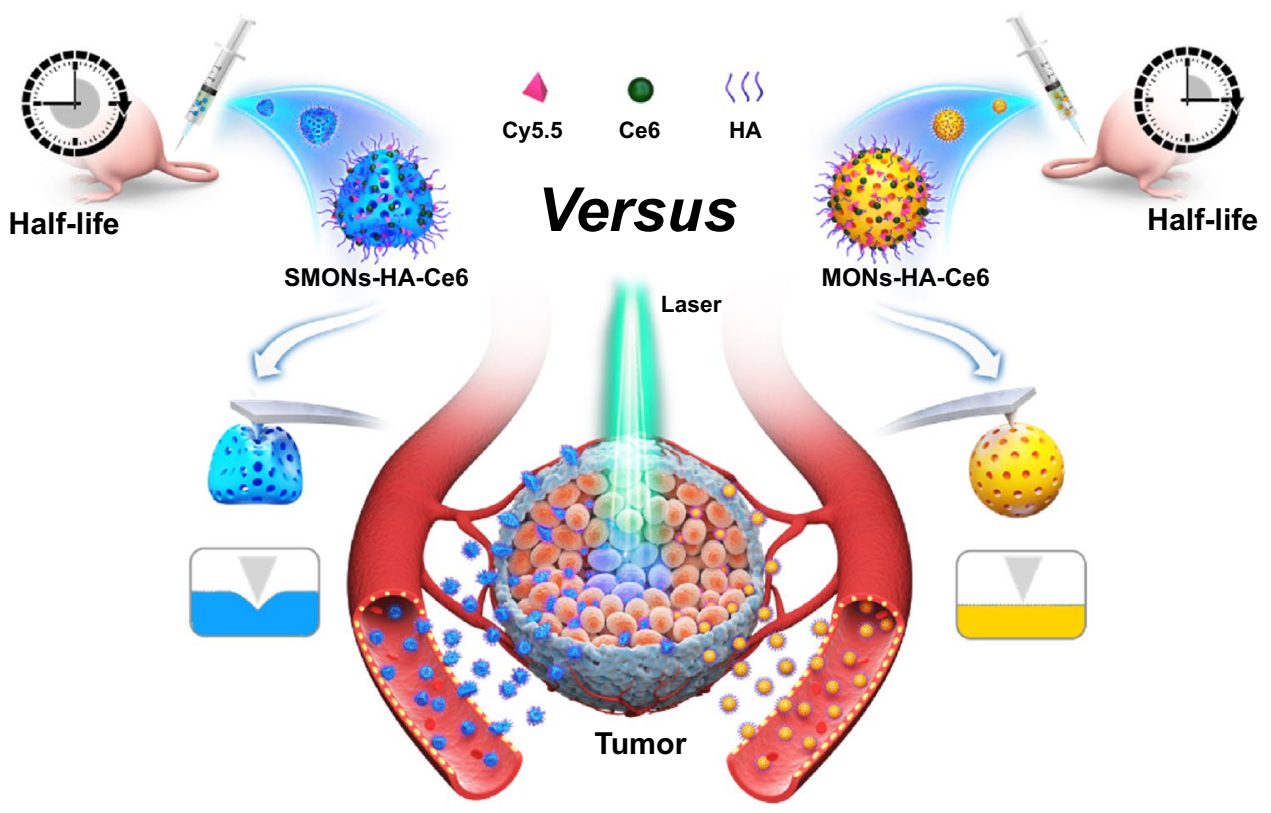

Scheme 1 Schematic of the soft nanoparticles as effective anticancer plateforms. The soft nanoplatforms exhibit enhanced tumor accumulation, extravasation from tumor vessels and penetration into deep tumor parenchyma and more efficient antitumor activity due to their softness and deformability compared with their stiff counterparts 
TEOS $(0.25 \mathrm{~mL})$ and TESPTS $(0.1 \mathrm{~mL})$ were added quickly to the mixture. The mixture was stirred at $35{ }^{\circ} \mathrm{C}$ for $24 \mathrm{~h}$ and then centrifuged resulting in the formation of thioetherbridged MONs, then rinsed thrice with ethanol.

\subsection{Modifying Amino Groups}

To graft amino groups on the MONs, the disulfide bonds that formed the thioether-bridged MONs were transformed to thiol groups through a reduction reaction and then covalently linked with $\mathrm{NH}_{2}$-maleimide. Briefly, $0.1 \mathrm{~g}$ of the MONs was dissolved in a mixture of $1.2 \mathrm{~mL}$ water, $4.4 \mathrm{~mL}$ dioxane, $0.20 \mathrm{~g}$ triphenylphosphine, and $80 \mu \mathrm{g} \mathrm{HCl}$ solution (37 $\mathrm{wt} \%$ ). After heating to $40{ }^{\circ} \mathrm{C}$ in an atmosphere of nitrogen for $2 \mathrm{~h}$, the thiol group linking the MONs was obtained by centrifugation of the other mixed solution, and washed thrice with water. Subsequently, the precipitation was dispersed

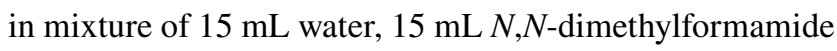
as well as $3.24 \mathrm{mg} \mathrm{NH}_{2}$-maleimide. After shaking for $12 \mathrm{~h}$ at room temperature, amino-modified MONs (denoted as MONs- $\mathrm{NH}_{2}$ ) were obtained by washing with water for three times.

\subsection{Preparation of MONs-HA and SMONs-HA}

MONs-HA were synthesized by coupling the MONs$\mathrm{NH}_{2}$ with carboxyl groups activated HA. Briefly, $0.1 \mathrm{~g}$ HA was first dissolved in $5 \mathrm{~mL}$ water. After adding NHS (20 $\left.\mathrm{mg} \mathrm{mL}^{-1}, 2.5 \mathrm{~mL}\right)$ and EDC $\left(20 \mathrm{mg} \mathrm{mL}^{-1}, 2.5 \mathrm{~mL}\right)$, this preparation was incubated at room temperature (RT) for $3 \mathrm{~h}$ while shaking. Thus, the carboxyl groups of HA were activated. Next, $0.08 \mathrm{~g}$ of $\mathrm{MONs}-\mathrm{NH}_{2}$ was mixed with the solution containing carboxyl groups activated HA, and the mixture was shaken for $24 \mathrm{~h}$. Then HA-modified MONs (denoted as MONs-HA) were obtained by centrifugation and washing thrice with water. Finally, the MONs-HA product without $\mathrm{CTAB}$ templates was obtained via three solvent extractions in ethanol $(300 \mathrm{~mL})$ at $27{ }^{\circ} \mathrm{C}$ for $12 \mathrm{~h}$. To obtain SMONs-HA, the MONs-HA $\left(2 \mathrm{mg} \mathrm{mL}^{-1}\right)$ were dispersed in $0.1 \mathrm{M} \mathrm{NaOH}$ aqueous solution for $15 \mathrm{~min}$. After washing with water, it was mixed with ethanol and stored until use.

\subsection{Preparation of MONs-HA-Cy5.5 and SMONs-HA-Cy5.5}

In brief, the MONs-HA (60 mg) and SMONs-HA $(20 \mathrm{mg})$ with the same number of particles (etched from the $60 \mathrm{mg}$ of MONs-HA) were, respectively, dispersed in a mixture (water $(12 \mathrm{~mL})$ Cy5.5-maleimide $(0.19 \mathrm{mg}), \mathrm{N}, \mathrm{N}$-dimethylformamide $(1.2 \mathrm{~mL})$ ). Following shaking for $12 \mathrm{~h}$ at RT, Cy5.5-modified nanoparticles were obtained (denoted as MONs-HA-Cy5.5 and SMONs-HA-Cy5.5, in the order) by centrifuging and washing with water.

\subsection{Preparation of SMONs-HA-Ce6 and MONs-HA-Ce6}

The carboxyl groups of Ce6 were activated by mixing Ce6 ( $1 \mathrm{mg} \mathrm{mL}^{-1}$ in $\mathrm{N}, \mathrm{N}$-dimethylformamide, $0.5 \mathrm{~mL}$ ), NHS $(1 \mathrm{mg})$, and EDC (1 mg) under shaking at RT for $3 \mathrm{~h}$. Then, $1 \mathrm{mg}$ of the MONs-HA-Cy5.5 or SMONs-HA-Cy5.5 was added. After shaking for $24 \mathrm{~h}$, the $\mathrm{Ce} 6$ grafted nanoparticles (denoted as MONs-HA-Ce6 and SMONs-HA-Ce6, respectively) were obtained by centrifuging and washing with water.

\subsection{Characterization}

A transmission electron microscope (TEM) was utilized to characterize the nanoparticles, with operation settings comprising $100 \mathrm{kV}$ on a Hitachi HT7700 microscope (Tokyo, Japan). The electron microscope (FEI Talos F200X) equipped with an energy-dispersive X-ray (EDX) was employed to obtain high-resolution transmission electron data and elemental mapping. Scanning electron microscopy (SEM) measurements were performed at $5 \mathrm{kV}$ (Hitachi S4800 microscope from Tokyo, Japan). Hydrodynamic size and zeta potential were measured using a ZetaPALS analyzer (Brookhaven Instruments Co., Holtsville, USA). An NEXUS870 spectrometer was employed to obtain Fourier transform infrared (FTIR) spectra. The Bruker AVIII400 spectrometer $(9.47 \mathrm{~T}, 79.48 \mathrm{MHz}$ of operating frequency, $6.0 \mathrm{kHz}$ of spin rate, and $120 \mathrm{~s}$ of recycle delay) was utilized to obtain the ${ }^{29} \mathrm{Si}$ magic-angle spinning (MAS) NMR spectra. A Micromeritics ASAP analyzer (2020) was employed 
to determine the Nitrogen adsorption-desorption isotherms at $-196{ }^{\circ} \mathrm{C}$. First, the materials were degassed at $150{ }^{\circ} \mathrm{C}$ under vacuum for $10 \mathrm{~h}$ or more. By using the adsorption data in a relative pressure $\left(p / p_{0}\right)$ range of 0.12 to 0.20 , we calculated the specific surface area (SBET) using the Brunauer-Emmett-Teller (BET) approach. We employed suitable nonlocal density functional theory (NLDFT) approaches to acquire pore-size distribution. Utilizing the amount adsorbed at a $p / p_{0}$ of 0.994 , we estimated the total pore volume. Young's modulus of the samples was estimated with the AFM in the mode of Peak Force Quantitative Nanomechanical Mapping (PeakForce QNM, Dimension Icon AFM, Bruker, U.S.A.) employing the TESPA-V2 AFM cantilever (Bruker AFM probes). After placing a drop of aqueous sample suspension on a clean silicon surface, the Hertzian model was used to calculate Young's modulus according to the slope in the linear region of the retraction curve. Infinite M200 PRO microplate scanner (Tecan, Switzerland) was used to record the intensity of the fluorescence.

\subsection{Cytotoxicity Assessment}

The cytotoxic MCF-7 cells in a Complete Medium DMEM solution and then cultured in a 96-well plate $(10,000$ cells in each well) for $12 \mathrm{~h}$. Next, we removed the supernatant and grown cells with $200 \mu \mathrm{L}$ of MONs-HA-Cy5.5 or SMONsHA-Cy5.5 at various concentrations $\left(25-400 \mu \mathrm{g} \mathrm{mL}^{-1}\right)$ for $24 \mathrm{~h}$. Then, $20 \mu \mathrm{L}$ of the CCK-8 solution was mixed. Finally, the absorbance $\left(\mathrm{OD}_{450 \mathrm{~nm}}\right)$ was read after $1 \mathrm{~h}$ with a microplate scanner. Cell viability (CV) was estimated as follows:

$\mathrm{CV}=\frac{A_{\text {sample }}-A_{(-) \text {control }}}{A_{(+) \text {control }}-A_{(-) \text {control }}}$.

\subsection{Hemocompatibility Assay}

The human blood sample was donated by a healthy young woman with the approval of the local medical ethics committee. In brief, $10 \mathrm{~mL}$ of normal saline (NS) were mixed with blood samples $(2 \mathrm{~mL})$, and centrifuged (2000 rpm, $5 \mathrm{~min}$ ) to isolate the red blood cells (RBC). The RBCs were washed using NS for several times. When the supernatant turned colorless, the RBCs were resuspended in $10 \mathrm{~mL}$ of NS. A mixture of $200 \mu \mathrm{L}$ of RBCs and $800 \mu \mathrm{L}$ of the
MONs-HA-Cy5.5 or SMONs-HA-Cy5.5 with different concentrations $\left(25-400 \mu \mathrm{g} \mathrm{mL}^{-1}\right)$ was prepared. To set negative or positive control, $(200 \mu \mathrm{L}) 800 \mu \mathrm{L}$ of NS or water was, respectively, mixed with RBC suspension. After maintaining at $37^{\circ} \mathrm{C}$ for $2 \mathrm{~h}$, the mixtures were centrifuged to get supernatant. A microplate reader was used to measure the concentration at OD of $570 \mathrm{~nm}$. Hemolysis $(\mathrm{H})$ was determined as follows:

$\mathrm{H}=\frac{\mathrm{OD}_{\text {sample }}-\mathrm{OD}_{(-) \text {control }}}{\mathrm{OD}_{(+) \text {control }}-\mathrm{OD}_{(-) \text {control }}}$.

\subsection{In Vivo Toxicity}

MONs-HA-Cy5.5 (100 $\mu \mathrm{L})$ and SMONs-HA-Cy5.5 (100 $\mu \mathrm{L}$ ) were injected respectively into male ICR mice (age; 4 weeks; $n=3 ; 20 \mathrm{mg} \mathrm{kg}^{-1}$ ). The mice were killed 21 days post-injection for harvesting of key organs (heart, liver, spleen, kidney, and lungs). Subsequently, the organs were subjected to hematoxylin and eosin (H\&E) staining assay. Finally, the sections were imaged using an optical microscope (IX71; Olympus, Tokyo, Japan).

\subsection{Cellular Uptake}

Human breast cancer MCF-7 cells were grown in 6-well plates $\left(1 \times 10^{6}\right.$ cells per well $)$ overnight. Subsequently, we substituted the medium in every well with $2 \mathrm{~mL}$ of fresh growth medium comprising MONs-HA-Cy5.5 or SMONs-HA-Cy5.5. The final concentration of MONs-HA-Cy5.5 and SMONs-HACy5.5 were 100 and $33 \mu \mathrm{g} \mathrm{mL}^{-1}$, respectively. The $33 \mu \mathrm{g} \mathrm{mL}^{-1}$ of SMONs-HA-Cy5.5 was obtained from $100 \mu \mathrm{g} \mathrm{mL}^{-1}$ of MONs-HA-Cy5.5 by etching approach, and thus they have the same number of particles. After culturing the cells for 1, 3, and $6 \mathrm{~h}$, they were digested and suspended in $200 \mu \mathrm{L}$ of PBS after washing with PBS for three times. The flow cytometry system was employed for quantification of cellular uptake of the two nanoplatforms. Six hours following incubation with MONs-HA-Cy5.5/SMONs-HA-Cy5.5, the cells were fixed for 20 min with $4 \%$ paraformaldehyde, then stained with DAPI. The intracellular association and nuclear morphology of the nanoplatforms were examined using confocal laser scanning microscopy (CLSM). The fluorescence intensity was analyzed using image-J software. 


\subsection{In Vivo Blood Circulation}

To evaluate the in vivo circulation behavior of the MONsHA-Cy5.5 and SMONs-HA-Cy5.5, BALB/c mice were injected with MONs-HA-Cy5.5 or SMONs-HA-Cy5.5 (100 $\left.\mu \mathrm{L}, 20 \mathrm{mg} \mathrm{kg}^{-1}\right)$ through the tail vein. Then the blood sample $(20 \mu \mathrm{L})$ was collected at different time points $(1,3,5,10,15$, $30 \mathrm{~min}$, and 1, 3, $6 \mathrm{~h}$ ) after injection. The blood was added into the anticoagulant tube, which contains NS (1 mL). Inductively coupled plasma (ICP) was used to measure the Si concentration. In vivo terminal half-life and other pharmacokinetic parameters of the MONs-HA-Cy5.5 or SMONs-HA-Cy5.5 was determined by a double-component pharmacokinetic model using DAS 3.0 (BioGuider Co., Shanghai, China).

\subsection{In Vivo Biodistribution}

MCF-7 tumors were subcutaneously injected $\left(2 \times 10^{7}\right.$ tumor cells) in the right forelimb of female BALB/c nude mice (age; 6 weeks). The amounts of the tumor in mice were checked daily until a volume of $200 \mathrm{~mm}^{3}$ was reached. To further identify the difference between the MONs-HA-Cy5.5 and SMONs-HA-Cy5.5 in terms of in vivo biodistribution, MONs-HA-Cy5.5 $\left(100 \mu \mathrm{L}, 20 \mathrm{mg} \mathrm{kg}^{-1}\right)$ or SMONs-HACy5.5 $\left(100 \mu \mathrm{L}, 6.67 \mathrm{mg} \mathrm{kg}^{-1}\right)$ with the same number of particles were administered intravenously into MCF-7 tumor-bearing $\mathrm{BALB} / \mathrm{c}$ mice $(n=3)$. Twenty-four hours after the treatment, we killed the mice and harvested the tumors plus major organs, which included: heart, spleen, liver, kidney, lung, and muscle tissues flanking the tumors. An IVIS Lumina XR system under a Cy5.5 filter $\left(\lambda_{\mathrm{ex}}=640 \mathrm{~nm}\right.$, $\lambda_{\mathrm{em}}=705 \mathrm{~nm}$ ) was employed to image the harvested specimens. To further investigate the long-term accumulating effect of the samples in each organ, the mice $(n=3)$ were killed at different times (including 3, 6, 12, 24, 48, and $72 \mathrm{~h}$ ) post-injection. Subsequently, their major organs were harvested and imaged as described above.

\subsection{Penetration in MCF-7 Tumor Spheroids}

To establish MCF-7 multicellular spheroids (MCSs) as an in vitro tumor model, MCF-7 cells grown in 96-well plates $(10,000$ cells per well] were precoated by $50 \mu \mathrm{L}$ of $1.5 \mathrm{w} / \mathrm{v} \%$ hot agarose solution and then cooled to RT) and cultured for 4 days at $37{ }^{\circ} \mathrm{C}$ to grow into spheroids. Then the MCSs were co-cultured with the MONs-HA-Cy5.5 $\left(100 \mu \mathrm{g} \mathrm{mL}^{-1}\right)$ or SMONs-HA-Cy5.5 $\left(33 \mu \mathrm{g} \mathrm{mL}^{-1}\right)$ with the same number of particles. Four hours later, the MCSs were harvested and rinsed with PBS to be examined using CLSM. The analysis of images was conducted using image-J.

\subsection{Intratumoral Distribution}

To study the intratumoral penetration of the MONs-HACy5.5 and SMONs-HA-Cy5.5, mice bearing MCF-7 xenografts were intravenously injected with MONs-HA-Cy5.5 $\left(100 \mu \mathrm{L}, 20 \mathrm{mg} \mathrm{kg}^{-1}\right)$ or SMONs-HA-Cy5.5 (100 $\mu \mathrm{L}$, $6.67 \mathrm{mg} \mathrm{kg}^{-1}$ ) with the same number of particles. The mice were killed $24 \mathrm{~h}$ later to extract the tumors, which were sectioned into slides of $8 \mu \mathrm{m}$ thickness in a cryostat. We stained the blood vessels following a standard protocol [38], and the tumor slices were then observed using CLSM.

\subsection{Detection of Reactive ${ }^{1} \mathrm{O}_{2}$}

Singlet oxygen was detected using SOSG. The intrinsic fluorescence of SOSG increases in the presence of ${ }^{1} \mathrm{O}_{2}$. Briefly, MONs-HA-Ce6 or SMONs-HA-Ce6 $\left(100 \mu \mathrm{L}, 1 \times 10^{-6} \mathrm{~m}\right.$ Ce6 equiv.) were mixed with SOSG $\left(10 \mu \mathrm{L}, 50 \times 10^{-6} \mathrm{M}\right)$ in $\mathrm{H}_{2} \mathrm{O}_{2}$ solution ( $3 \mathrm{wt} \%$ ). Then, the mixture was irradiated by a 660 -nm laser $\left(0.5 \mathrm{~W} \mathrm{~cm}^{-2}\right)$ for different time $(0,1$, $3,5,10 \mathrm{~min})$. Finally, a microplate reader $\left(\lambda_{\mathrm{ex}}=490 \mathrm{~nm}\right.$, $\lambda_{\mathrm{em}}=530 \mathrm{~nm}$ ) was used to measure the intensity of the fluorescence.

\subsection{Determining Reactive Oxygen Species (ROS) in Cells}

DCFH-DA was employed to evaluate the accumulation of total ROS in the cells. The total ROS was indicated by fluorescence intensity adducts of DCFH-DA [i.e., dichlorofluorescein (DCF)]. Briefly, MCF-7 cells $\left(1 \times 10^{5}\right.$ cells/well) were incubated in 96-well plate in DMEM (100 $\mu \mathrm{L})$ supplemented with $10 \%$ FBS and cultured overnight. Next, we co-incubated the cells with Ce6, MONs-HA-Ce6, or SMONs-HA-Ce6 (100 $\mu \mathrm{L}, 4 \times 10^{-6} \mathrm{M} \mathrm{Ce} 6$ equiv.) for $6 \mathrm{~h}$. The control group was set by adding $100 \mu \mathrm{L}$ of PBS. The media were then replaced by fresh DMEM $(100 \mu \mathrm{L})$ supplemented with DCFH-DA $\left(20 \times 10^{-6} \mathrm{M}\right)$ followed 
by a further $4 \mathrm{~h}$ incubation. Next, we washed the cells with PBS and subjected them to 660-nm laser irradiation $\left(0.5 \mathrm{~W} \mathrm{~cm}^{-2}\right)$ for $5 \mathrm{~min}$. Finally, a microplate reader $\left(\lambda_{\mathrm{ex}}=485 \mathrm{~nm}, \lambda_{\mathrm{em}}=525 \mathrm{~nm}\right)$ was employed to record the fluorescence intensity.

\subsection{In Vitro PDT}

MCF-7 cells $\left(1 \times 10^{5}\right.$ cells/well $)$ were seeded into a 96-well plate and cultured for $12 \mathrm{~h}$. After that, we co-incubated the cells for $24 \mathrm{~h}$ with $200 \mu \mathrm{L}$ of Ce6, MONs-HA$\mathrm{Ce} 6$, or SMONs-HA-Ce6 at various $\mathrm{Ce} 6$ concentrations (1-4 $\times 10^{-6} \mathrm{M}$ Ce6 equiv.). Next, the cells were subjected to a $660-\mathrm{nm}$ laser $\left(0.5 \mathrm{~W} \mathrm{~cm}^{-2}\right)$ radiation for $5 \mathrm{~min}$. The experiments were repeated three times. The therapeutic effects were analyzed using the CCK8 assay.

\subsection{In Vivo Antitumor Effect}

BALB/c mice inoculated with MCF-7 tumors ( $n=5$, each group) were grouped into seven groups: (I) PBS group, (II) MONs-HA-Ce6 group, (III) SMONs-HA-Ce6 group, (IV) PBS + laser group, (V) free Ce6 + laser group, (VI) MONsHA-Ce6 + laser group, (VII) SMONs-HA-Ce6 + laser group. Subsequently, we injected $100 \mu \mathrm{L}$ of each material intravenously (The relevant dosage of $\mathrm{Ce} 6$ of each group was approximately $6 \mathrm{mg} \mathrm{kg}^{-1}$ except the PBS group). Twentyfour hours following injection, we subjected the mice to 660$\mathrm{nm}$ laser radiation $\left(0.5 \mathrm{~W} \mathrm{~cm}^{-2}, 10 \mathrm{~min}\right)$. Four mice per group were killed at $24 \mathrm{~h}$ post-irradiation, and the collected tumors were stained with hematoxylin and eosin (H\&E) for histopathology assessment. We recorded the tumor volumes and body weights for 14 days consecutively. The equation below was used to calculate the tumor volume $(\mathrm{V})$ : $\mathrm{V}=\mathrm{Width}^{2} \times$ length $/ 2$. The volume of tumor after therapy was divided by the volume before therapy to obtain the relative tumor volume $\left(V / V_{0} ; V_{0}\right.$ is tumor volume pretherapy). On the last day of the experiment, we killed the mice and took photographs and weights of the tumors. Approval for conducting these experiments was obtained from the Animal Care and Use Committee of Jinling Hospital.

\subsection{Data Analysis}

Experimental were processed using GraphPad Software (CA, USA) and are shown as mean \pm standard deviation. Results of two groups were compared using student's $t$ test, whereas multiple groups were compared using the variance (ANOVA) test. Significance was set a $P<0.05$.

\section{Results and Discussion}

\subsection{Characterization of SMONs-HA-Cy5.5 and MONs-HA-Cy5.5}

The CTAB-directed solgel process was utilized to prepare thioether-bridged MONs and then covalently modified with $\mathrm{HA}$ and $\mathrm{Cy} 5.5$ after reducing thioether moieties and connecting with $\mathrm{NH}_{2}$-maleimide. The TEM and SEM images indicated that both the MONs and MONs-HACy5.5 possess a well-defined spherical shape with an identically uniform diameter of $180 \mathrm{~nm}$ (Figs. 1a-c and S1). The soft SMONs-HA were prepared using a preferential etching approach. After being etched in an aqueous $\mathrm{NaOH}$ solution $(0.1 \mathrm{M})$ for $15 \mathrm{~min}$, the surface of the MONs-HA collapsed and uniformly shrank inward, transforming into a cross-wrinkled morphology. According to the SEN and TEM images, the SMONs-HA-Cy5.5 have a hollow structure (Fig. 1d-f). The shell thickness, diameter, and cavity size of the SMONs-HA-Cy5.5 are estimated to be 24, 205, and $160 \mathrm{~nm}$, respectively. STEM-HAADF images further confirm the symmetrically cross-wrinkled morphology and the ultrathin shell (Fig. 1g). EDX elemental mapping of the SMONs-HA-Cy5.5 indicated that C, Si, S, and O are mainly distributed on the outside ridge of the hollow structure, while $\mathrm{C}, \mathrm{Si}, \mathrm{S}$, and $\mathrm{O}$ are homogeneously deposit in the whole solid sphere before etching (Figs. $1 \mathrm{~h}-\mathrm{i}$ and S2). The mechanism of preparing SMONs from MONs has been reported in our previous work. Directly speaking, the organosilica nanospheres have a more stable surface with highly cross-linked $\mathrm{Si}-\mathrm{OH}$ groups via ammonia catalysis in the sol-gel process directed by CTAB. The less condensed cores of the MONs-HA and the inorganic silica species in the shell are preferentially etched away 

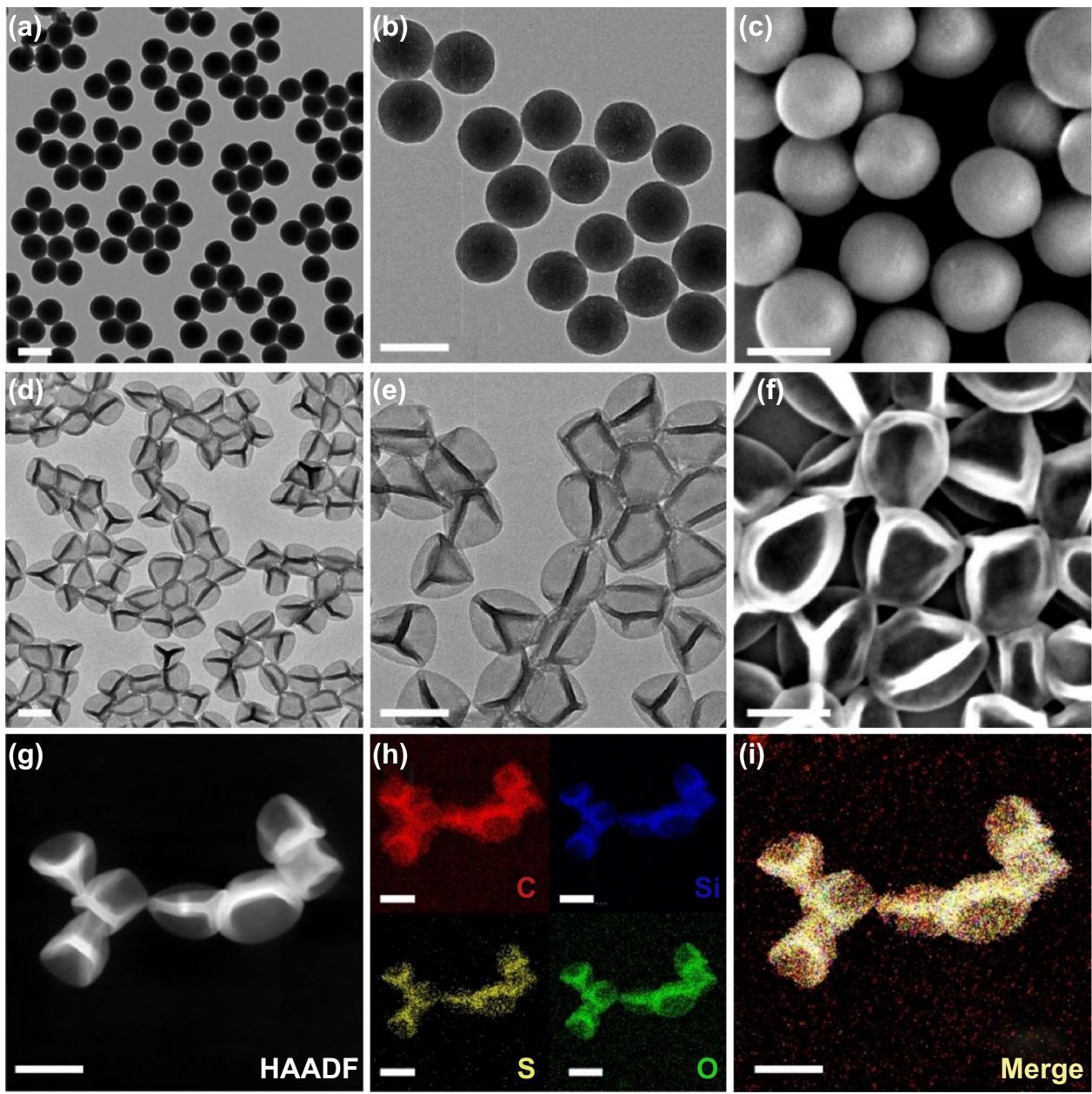

Fig. 1 a, b TEM and $\mathbf{c}$ SEM images of the MONs-HA-Cy5.5. d, e TEM and $\mathbf{f}$ SEM images of the SMONs-HA-Cy5.5. g STEM-HAADF image and $\mathbf{h}$, i EDX elemental mapping images of the SMONs-HA-Cy5.5. Red, blue, yellow, and green indicate the elements $\mathrm{C}, \mathrm{Si}, \mathrm{S}$, and $\mathrm{O}$, respectively. Scale bars, $200 \mathrm{~nm}$. (Color figure online)

by $\mathrm{OH}^{-}$ions when dispersed in $\mathrm{NaOH}$ solution, resulting in significantly increased ratio of flexible $\mathrm{Si}-\mathrm{R}-\mathrm{Si}$ chains, and noncross-linked free $\mathrm{Si}-\mathrm{OX}$ groups in holistic frameworks. The shell becomes thinner and deform inward via internal van der Waals force, which eventually results in the cross-linked and coarse morphology of SMONs-HA. More importantly, the dominating free $\mathrm{Si}-\mathrm{OX}$ groups in incomplete condensation and flexible $\mathrm{Si}-\mathrm{R}-\mathrm{Si}$ chains, as well as the hollow nanostructure with ultrathin shells amid the frameworks, grant SMONs-HA the unique soft character.

The hydrodynamic diameter, zeta potential, chemical composition, and mesoporous structure of the soft SMONsHA and the hard counterparts were evaluated. The hydrodynamic diameters of the MONs, MONs- $\mathrm{NH}_{2}$, MONs-HA and SMONs-HA are measured to be about 181, 184, 251, and $268 \mathrm{~nm}$, respectively (Fig. 2a). Both the MONs-HA and SMONs-HA have a narrow polydispersity index (PDI): $0.106 \pm 0.01$ and $0.139 \pm 0.02$, suggesting their excellent dispersity in aqueous solutions. The zeta potential of the MONs, MONs-NH $\mathrm{N}_{2}$, MONs-HA and SMONs-HA are measured to be $33.0 \pm 0.2,35.4 \pm 1.7,-33.8 \pm 0.6$, and $-31.4 \pm 1.2 \mathrm{mV}$, respectively (Fig. 2b). The negatively charged surfaces for the MONs-HA and SMONs-HA demonstrate the successful modification of HA. Notably, there is no significant fluctuation in surface charge after the MONsHA were etched, suggesting the stability of the modified HA. Furthermore, the FTIR spectrum of the MONs exhibits C-S absorbance band at $691 \mathrm{~cm}^{-1}$, indicating the thioether group-incorporated frameworks (Fig. 2c) [39]. Clearly, both 

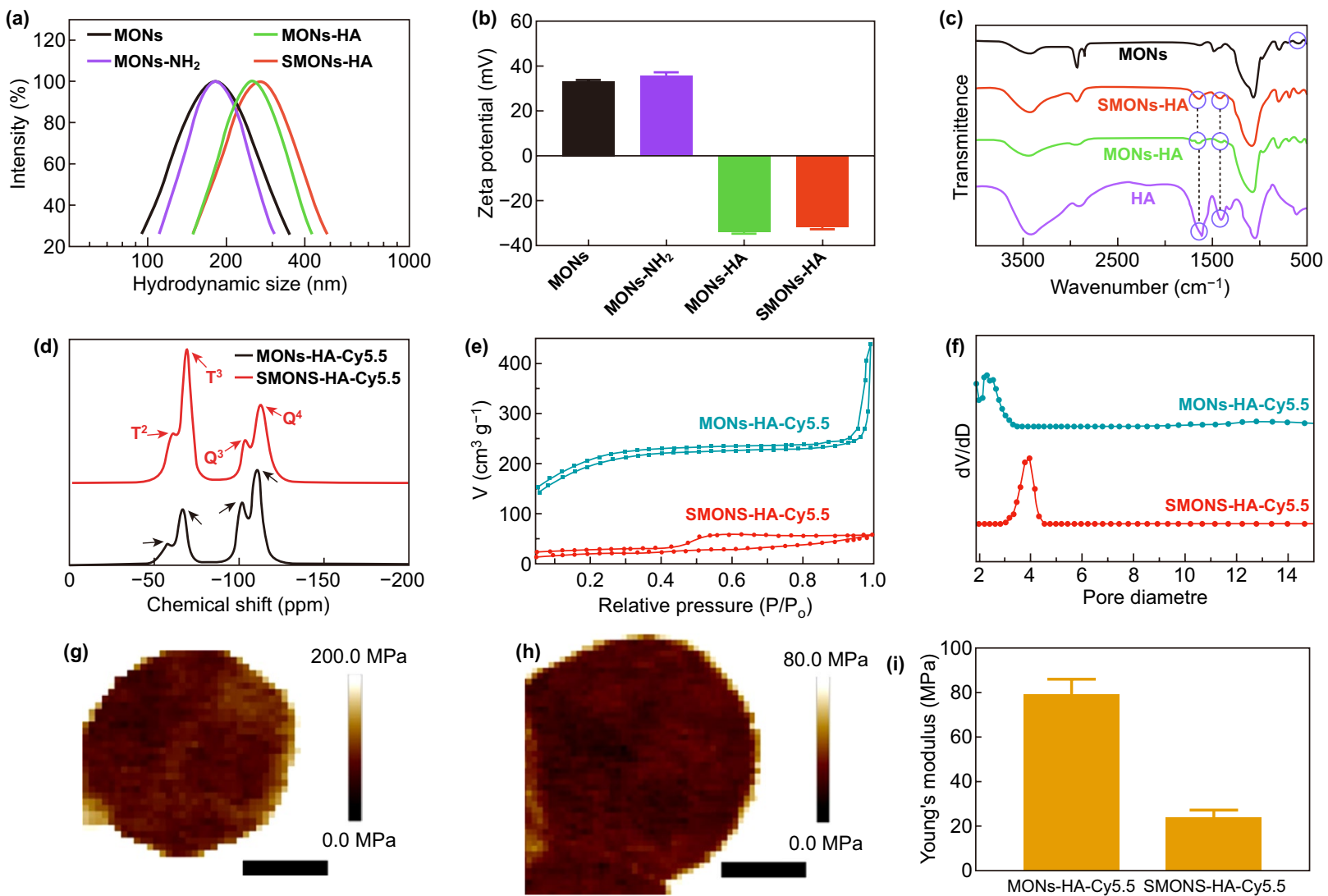

Fig. 2 a Hydrodynamic diameter and b zeta potential of the MONs, MONs-NH 2 , MONs-HA, and SMONs-HA. c FTIR spectrum of the MONs, HA, MONs-HA, and SMONs-HA. d ${ }^{29}$ Si MAS NMR spectra, e nitrogen sorption isotherms, $\mathbf{f}$ pore-size distribution curves, $\mathbf{g}$, $\mathbf{h}$ AFM images and $\mathbf{i}$ Young's modulus of the MONs-HA-Cy5.5 and SMONs-HA-Cy5.5. Scale bars, $100 \mathrm{~nm}$

SMONs-HA and MONs-HA show the absorbance bands at 1630 and $1380 \mathrm{~cm}^{-1}$ which are assigned to the carboxylate asymmetric and symmetric stretching vibration of the HA skeleton, respectively [40]. These results clearly illustrate the successful modification of HA on both MONs-HA and SMONs-HA. Furthermore, to make the materials detectable, a near-infrared fluorescent dye Cy5.5 was modified on the nanoplatforms, and the fluorescence intensities of the soft SMONs-HA-Cy5.5 and MONs-HA-Cy5.5 were modulated to similar values (Fig. S3). The 29Si MAS NMR spectra of SMONs-HA-Cy5.5 and MONs-HA-Cy5.5 both exhibited signals at $-110,-58,-102$, and $-67 \mathrm{ppm}$, respective to $Q^{4}\left(\mathrm{Si}(\mathrm{OSi})_{4}\right), Q^{3}\left(\mathrm{Si}(\mathrm{OSi})_{3}(\mathrm{OX})\right), T^{3}\left(\mathrm{C}-\mathrm{Si}(\mathrm{OSi})_{3}\right)$, and $T^{2}$ $\left(\mathrm{C}-\mathrm{Si}(\mathrm{OSi})_{2}(\mathrm{OX})\right)$ species, in that order (Fig. 2d). The presence of the $T$ peak reveals the organic group-incorporated frameworks. The $T^{n} /\left(T^{n}+Q^{m}\right)$ ratio of the SMONs-HACy5.5 is 0.604, which is remarkably higher in comparison with the MONs-HA-Cy5.5 (0.326), demonstrating that Q silicate species in frameworks are preferentially etched in $\mathrm{NaOH}$ aqueous solution. Relatively higher organic group content remained in the frameworks of SMONs-HA-Cy5.5 are favorable to the soft structure and deformability. The nitrogen sorption isotherms of the SMONs-HA-Cy5.5 and MONs-HA-Cy5.5 were further measured, which show type IV curves (Fig. 2e), indicating typical mesoporous structures. The comparatively large hysteresis loop of the SMONs-HACy5.5 suggests the appearance of hollow cavities. The surface areas of the SMONs-HA-Cy5.5 and MONs-HA-Cy5.5 are estimated to be 80.8 , and $495.6 \mathrm{~m}^{2} \mathrm{~g}^{-1}$, respectively, and their pore volumes are 0.086 and $0.416 \mathrm{~cm}^{3} \mathrm{~g}^{-1}$, respectively. Also, the pore sizes of the SMONs-HA-Cy5.5 and MONs-HA-Cy5.5 are calculated to be $4.0 \mathrm{~nm}$ and $2.3 \mathrm{~nm}$ based on the NLDFT method, respectively (Fig. 2f). Furthermore, the elastic Young's modulus $\left(E_{\mathrm{y}}\right)$ of the SMONsHA-Cy5.5 is measured to be $24.2 \pm 3.2 \mathrm{MPa}$ by using atomic force microscopy (AFM), which is about $31 \%$ of the $E_{\mathrm{y}}$ of 
their hard counterparts $(79.2 \pm 7.0 \mathrm{MPa}$, Fig. $2 \mathrm{~g}-\mathrm{i})$, clearly certifying the SMONs-HA-Cy5.5 are significantly "softer" than the MONs-HA-Cy5.5. Additionally, the diameter of the SMONs-HA-Cy5.5 is slightly larger than that of MONsHA-Cy5.5 when observed using AFM, which is most likely because that the SMONs-HA-Cy5.5 were squashed more significantly under compression during the testing process owing to its softer characteristic comparing with the hard ones. These results indicate that soft mesoporous organosilica nanoplatforms SMONs-HA-Cy5.5 and the hard MONs-HA-Cy5.5 with similar size, surface charge, component, fluorescence intensity have been obtained successfully. The hydrodynamic diameters of SMONs-HA-Cy5.5 and MONs-HA-Cy5.5 remain notably stable after storing in different solvent conditions for 2 weeks, indicating these nanoparticles are remarkably steady (Table S1). The hydrodynamic diameters and zeta potential of MONs-HA-Cy5.5 and SMONs-HA-Cy5.5 are measured to be 237.8, 249.2, - 15.6, and $-12.5 \mathrm{mV}$ after been stored in PBS aqueous solution for 2 weeks (Table S1 and Fig. S4). The value of zeta potential in PBS is lower than that in water, which is mainly because that the counter ions in PBS solution accumulate on the surface of nanoparticle, shielding the surface charge and thus reducing the absolute value of zeta potential. But the hydrodynamic diameters of MONs-HA-Cy5.5 and SMONs-HA-Cy5.5 measured in PBS solution stay similar with that measured in water due to the sterically stabilizing effect of HA. The results indicate that MONs-HA-Cy5.5 and SMONs-HA-Cy5.5 could be stored in PBS aqueous solution for at least 2 weeks and keep excellent dispersity.

\subsection{Biocompatibility}

To qualify the following biomedical applications, the cytotoxicity of the SMONs-HA-Cy5.5 and MONs-HACy5.5 to MCF-7 cells were examined. The cell viabilities remain over $80 \%$ even treated with SMONs-HA-Cy5.5 or MONs-HA-Cy5.5 at a concentration of up to $400 \mu \mathrm{gLL}^{-1}$ for $24 \mathrm{~h}$, and the hemolytic activities of both the SMONsHA-Cy5.5 and MONs-HA-Cy5.5 are lower than $1 \%$ at a concentration of up to $400 \mu \mathrm{g} \mathrm{mL}^{-1}$, indicating excellent hemocompatibility (Fig. S5). The in vivo biocompatibility was further evaluated through hematoxylin and eosin (H\&E) staining, blood routine, and serum biochemical examinations. At 21 days after intravenous injection of the
SMONs-HA-Cy5.5 or MONs-HA-Cy5.5 at the dosages of $20 \mathrm{mg} \mathrm{kg}^{-1}$, the complete blood counts and serum chemistries show that no significant changes happen compared with normal saline injected controls. All parameters including blood cells (WBCs), red blood cells (RBCs), platelet (PLT), alanine aminotransferase (ALT), aspartate aminotransferase (AST), alkaline phosphatase (ALP), uric acid (UA), and direct bilirubin (D-BLL) are within normal reference ranges (Fig. S6a-h), indicating problems like myelosuppression, hemolysis, inflammation, or liver and kidney injury will not be caused by intravenous injection of the materials. Besides, there is no obvious inflammation or necrosis of major organs (heart, liver, spleen, lung, and kidney) in the H\&E staining images (Fig. S6i), which further indicates the excellent in vivo safety of the SMONs-HA-Cy5.5 and MONs-HA-Cy5.5.

\subsection{In Vitro Cellular Uptake}

The tumor cellular uptake of the SMONs-HA-Cy5.5 and MONs-HA-Cy5.5 was investigated using MCF-7 cells. The CLSM images show that the fluorescence signals are significantly stronger when the cells were incubated with the SMONs-HA-Cy5.5 (Figs. 3a and S7,). The relative intracellular Cy5.5 fluorescence intensity of MCF-7 cells incubated with the SMONs-HA-Cy5.5 for 3 or $6 \mathrm{~h}$ is about 1.7 times higher than that of MONs-HA-Cy5.5 group (Figs. 3b and S8). Furthermore, the cellular uptake of the SMONs-HACy5.5 and the MONs-HA-Cy5.5 was quantitatively investigated using flow cytometry. The percentages of cells associated with MONs-HA-Cy5.5 are measured to be $0.91 \%$, $3.84 \%$, and $23.85 \%$ after incubation with MONs-HA-Cy5.5 for 1,3 , and $6 \mathrm{~h}$, respectively (Fig. 3c, d). As for SMONsHA-Cy5.5 group, the numbers increase to $0.98 \%, 7.41 \%$, and $40.96 \%$ at the matched time intervals. The cellular uptake of SMONs-HA-Cy5.5 is almost twice that of their stiff counterparts after incubation with MCF-7 cell for $6 \mathrm{~h}$. In addition, the relative average fluorescence intensities of SMONs-HACy5.5 treated group are measured to be 1668 and 2159 after incubation for 3 and $6 \mathrm{~h}$ (Fig. 3e), both of which are up to 2-fold higher than that of the rigid MONs-HA-Cy5.5 group (852 and 1237). These findings strongly certify that the soft SMONs-HA-Cy5.5 nanoplatforms are more efficiently internalized by MCF-7 cells than the stiff ones. It is believed that the deformability of soft nanoparticles can 
(a)
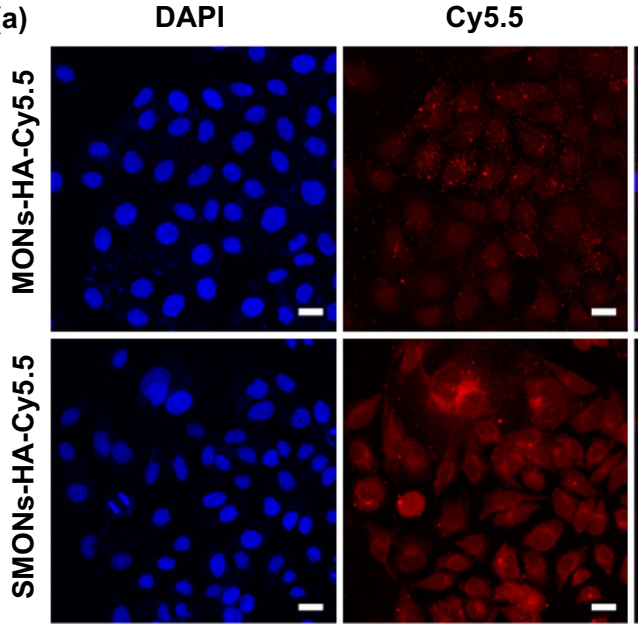

(c)
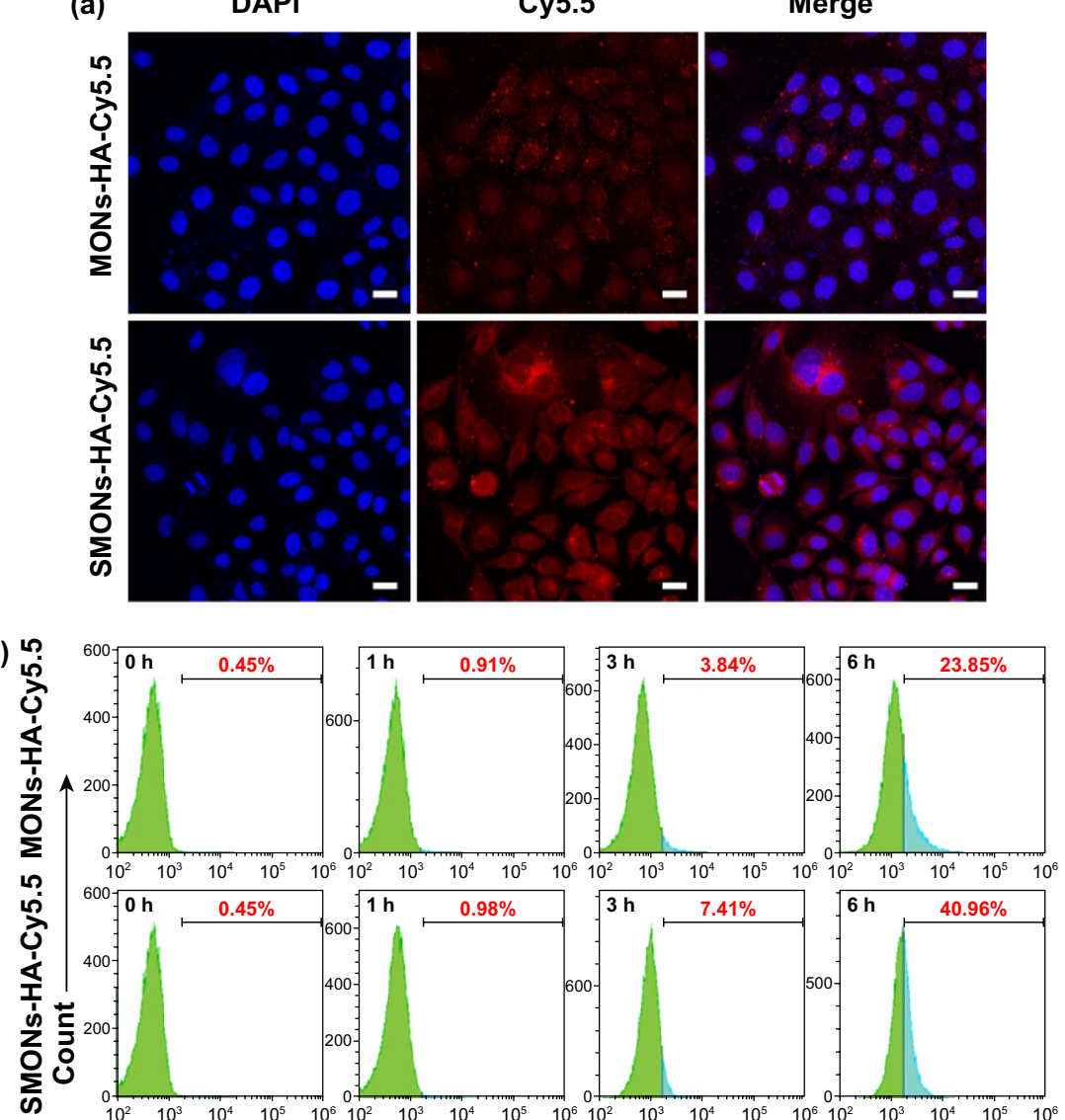

交|
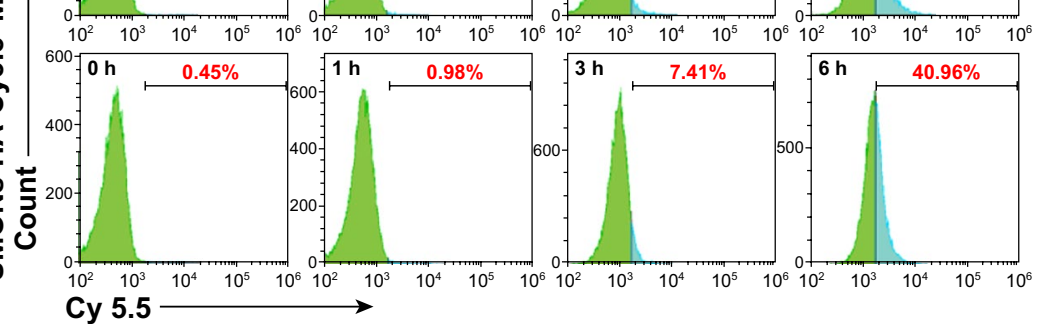

Cy 5.5

Fig. 3 Cellular uptake. a CLSM images and b relative fluorescence intensity analysis of MCF-7 cells incubated with the MONs-HA-Cy5.5 and SMONs-HA-Cy5.5 for $6 \mathrm{~h}$. Scale bars, $25 \mu \mathrm{m}$. $\mathbf{c}$ Flow cytometry, d relative cellular uptake efficiency and e mean fluorescence intensity of MCF-7 cells incubated with the MONs-HA-Cy5.5 and SMONs-HA-Cy5.5 for 1, 3, $6 \mathrm{~h}$

decrease the invagination of cellular membranes and thus save the corresponding energy needed for the wrapping of nanoparticles during internalization [37].

\subsection{Blood Circulation}

The in vivo application of nanoparticle (NP)-based drugs has been limited by their short circulation half-life [41]. To explore whether the soft nanoplatforms have advantages in pharmacokinetics, the SMONs-HA-Cy5.5 and MONsHA-Cy5.5 were separately injected into ICR mouse via tail vein. The circulation profiles were then obtained by measuring the Si concentration in blood samples (Fig. 4a). There is a sharp decline in the first $0.5 \mathrm{~h}$ after dosing in the concentration-time curves, indicating a distribution phase: the concentrations of SMONs-HA-Cy5.5 and
MONs-HA-Cy5.5 drop to $41.1 \%$ and $11.5 \%$ of injection dose, respectively. In the late elimination phase, the SMONs-HA-Cy5.5 group exhibits approximately 5-fold higher blood drug concentration compared with the MONs-HA-Cy5.5 group. At $6 \mathrm{~h}$ after intravenous injection, the remaining levels of SMONs-HA-Cy5.5 and MONs-HA-Cy5.5 in blood are measured to be $25.5 \%$ and $4.7 \%$. The pharmacokinetic analysis demonstrates that the corresponding clearance (CL) is 0.0143 for the SMONsHA-Cy5.5 and $0.0727 \mathrm{~L} \mathrm{~h}^{-1} \mathrm{~kg}^{-1}$ for MONs-HA-Cy5.5 (Fig. 4b). The significantly decreased CL for the SMONsHA-Cy5.5 indicates that the soft nanoplatforms provide a slower clearance from the circulation and a prolonged blood retention. Additionally, the terminal phase halflife of the SMONs-HA-Cy5.5 is 8.49 h (Fig. 4c), nearly 3 times longer than that of MONs-HA-Cy5.5 (3.07 h). 

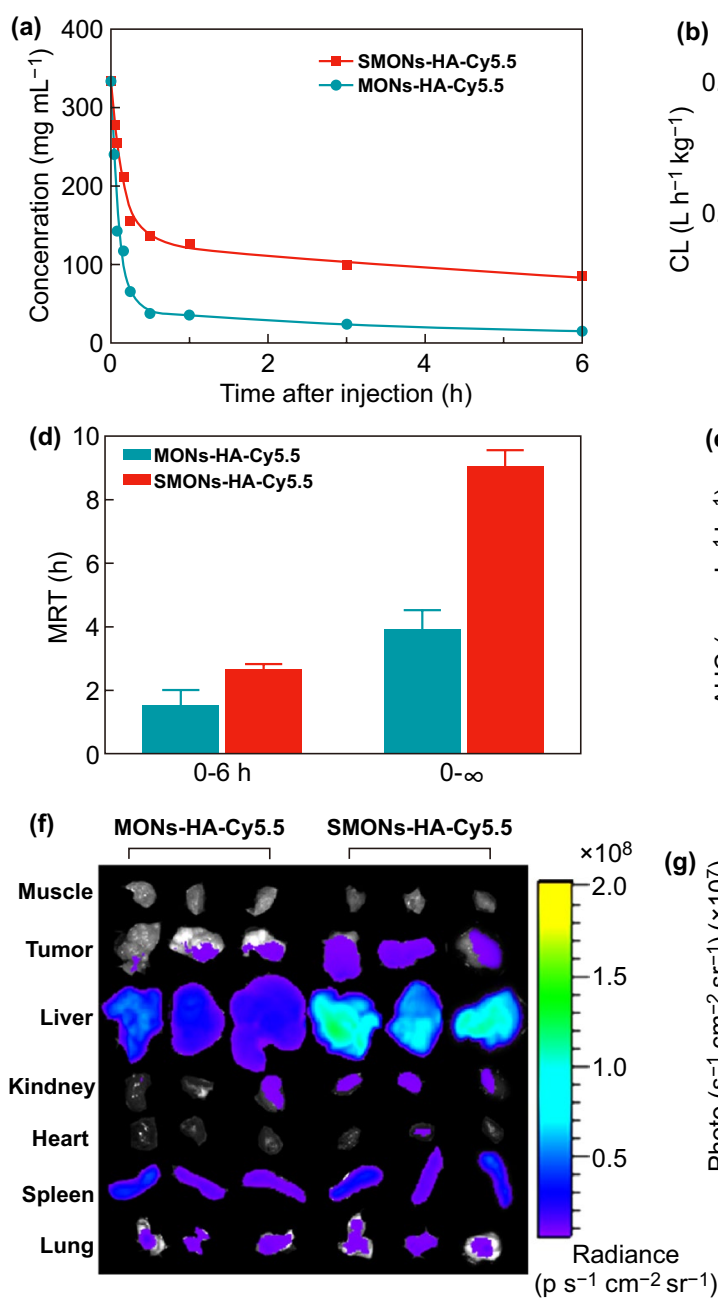
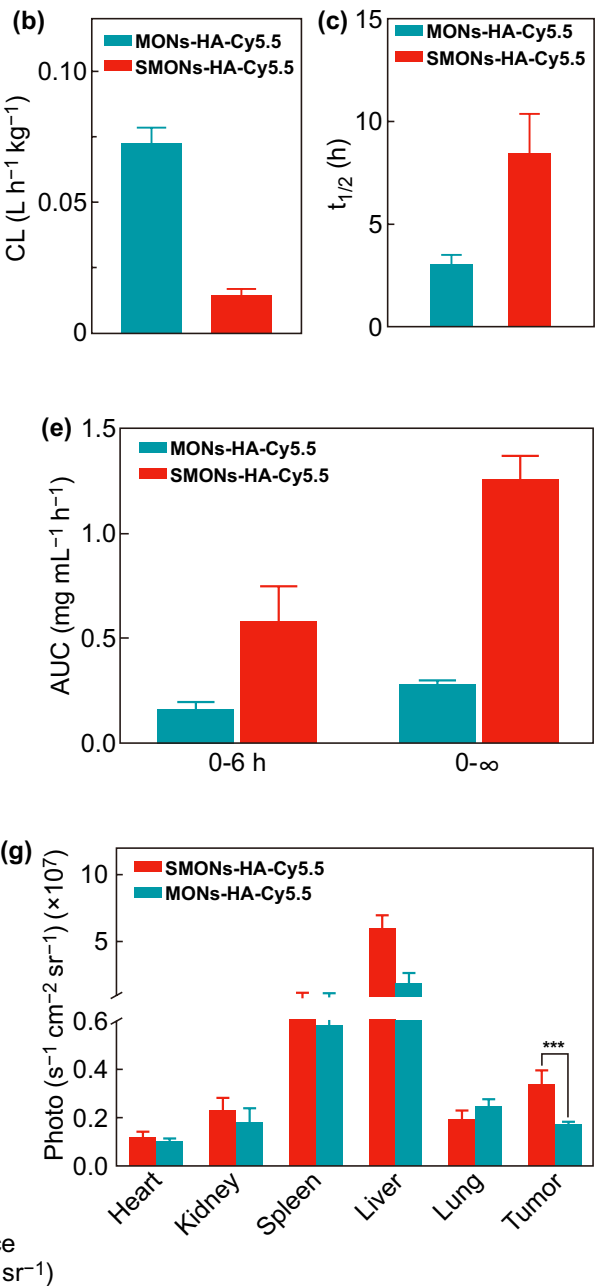

Fig. 4 Blood circulation and tumor accumulation. a Concentrations of Si in the blood at different time intervals after injection of MONs-HACy5.5 and SMONs-HA-Cy5.5. b The corresponding clearance (CL), $\mathbf{c}$ the terminal phase half-life $\left(t_{1 / 2}\right)$, $\mathbf{d}$ the mean residence time (MRT) and e blood concentration versus time curve (AUC) of MONs-HA-Cy5.5 and SMONs-HA-Cy5.5 by fitting the circulation profile. $\mathbf{f}$ Near-infrared fluorescence (NIFR) imaging and $\mathbf{g}$ quantification of the fluorescence intensity in the major organ and tumor from MCF-7 bearing mice $(n=3)$ at post-24 $\mathrm{h}$ after the administration of the SMONs-HA-Cy5.5 or MONs-HA-Cy5.5. $* * * P<0.001$

Furthermore, the mean residence time (MRT) and correlative area under the blood concentration versus time curve (AUC) were also calculated using a two-compartment model. $\mathrm{MRT}_{0-6 \mathrm{~h}}$ and $\mathrm{MRT}_{0-\infty}$ of the SMONsHA-Cy5.5 are 3.94 and $9.09 \mathrm{~h}$, whereas the numbers for MONs-HA-Cy5.5 are 1.45 and 2.67 h (Fig. 4d). AUC $0-6 \mathrm{~h}$ and $\mathrm{AUC}_{0-\infty}$ of the SMONs-HA-Cy5.5 are 0.582 and $1.258 \mathrm{mg} \mathrm{mL}^{-1} \mathrm{~h}^{-1}$, while the corresponding values for the MONs-HA-Cy5.5 are 0.165 and $0.276 \mathrm{mg} \mathrm{mL}^{-1} \mathrm{~h}^{-1}$ (Fig. 4e). Notably, the SMONs-HA-Cy5.5 show significant advantages (3-4 fold) over the stiff counterparts in terms of these pharmacokinetic parameters. These results clearly demonstrate that the soft nanoplatforms display a longer blood circulation in vivo than the hard ones. The possible explanation for the prolonged circulation time of deformable nanoplatforms is that they have unique fluid mechanics compared to stiff ones in the flowing bloodstream $[42,43]$.

\subsection{In Vivo Biodistribution}

Since long circulation time in vivo is strikingly essential for nanomaterials to accumulate in solid tumors via the EPR effect, the accumulation of the SMONs-HA-Cy5.5 
and MONs-HA-Cy5.5 in tumors was further evaluated in MCF-7 tumor-bearing BALB/c nude mice. The fluorescence intensities of the SMONs-HA-Cy5.5 and MONs-HA-Cy5.5 solutions were very close (Fig. S9). At 24 h post-injection, the mice were killed, and their tumors and major organs were collected and subjected to the IVIS Lumina $X R$ system. Notably, the tumors of the mice injected with the MONsHA-Cy5.5 show stronger fluorescence than those injected with MONs-HA-Cy5.5 (Fig. 4f). The average fluorescence intensity of the tumors in the SMONs-HA-Cy5.5 group is $0.34 \times 10^{7}$ photo $\mathrm{s}^{-1} \mathrm{~cm}^{-2} \mathrm{sr}^{-1}$, twice higher than that of MONs-HA-Cy5.5 group $\left(0.17 \times 10^{7}\right.$ photo s$\left.{ }^{-1} \mathrm{~cm}^{-2} \mathrm{sr}^{-1}\right)$ at $24 \mathrm{~h}$ after intravenous injection (Fig. 4g). These results demonstrate that tumor accumulation of soft SMONs-HA-Cy5.5 is significantly higher than that of the rigid counterparts, indicating that the soft structure of the SMONs-HA-Cy5.5 is indeed conducive to their accumulation in tumor sites, which is related to its prolonged circulation time and more efficient cellular uptake. It is noted that although the accumulation of SMONs-HA-Cy5.5 in tumors is higher than that of MONs-HA-Cy5.5, both of them are still low considering the accumulation in the liver or spleen. It is due to the fact that the liver and spleen are major biological barriers that prevent drug delivery to solid tumors because they sequester the majority of administered nanomaterials [44, 45]. Thus, more elaborated modifications are needed in their future application. For example, coat the soft nanoplatforms with a dense and dynamic outer PEG layer or cell-membrane components to cloak the particles from liver endothelial cells $[15,16]$, modify tumor-targeting ligands on the surface of nanoparticles to further enhance their accumulation in tumors $[42,46]$. A series of ex vivo images of mice over $72 \mathrm{~h}$ show that fluorescence signals are mainly concentrated in the liver and spleen, and the intensity of fluorescence signals decrease gradually with the prolongation of injection time (Fig. S10). The results indicate that most of the nanoparticles are engulfed by the reticuloendothelial system and metabolized through the liver and spleen. Interestingly, during the initial period after injection, the soft SMONsHA-Cy5.5 accumulate in the liver and spleen more rapidly than MONs-HA-Cy5.5. However, with the time going on, the accumulation of liver and spleen of soft SMONs-HACy5.5 group and MONs-HA-Cy5.5 tends to be the similar after $24 \mathrm{~h}$ post-injection.

\subsection{Tumor Penetration in Multicellular Spheroids (MCSs)}

It is speculated that soft nanoparticles could deform and squeeze into ECM when they contact the surface of tumor. Herein, multicellular spheroids derived from MCF-7 cells were employed as in vitro $3 \mathrm{D}$ models to investigate the discrepancy in tumor penetration capacity between the SMONs-HA-Cy5.5 and the stiff counterparts. After coincubated with SMONs-HA-Cy5.5 or MONs-HA-Cy5.5 for $4 \mathrm{~h}$, the MCSs were subjected to CLSM Z-stack scanning. The outer edge of MCSs was defined as $0 \mu \mathrm{m}$. The CLSM images show that the fluorescence signals are stronger and penetrate deeper through the distance from 30 to $90 \mu \mathrm{m}$ in SMONs-HA-Cy5.5 treated group, whereas the fluorescence signals of the rigid MONs-HA-Cy5.5 group are relatively weaker and mainly distribute in the peripheral area of MCSs (Fig. 5a). For example, at the scanning depth of $30 \mu \mathrm{m}$, the fluorescence intensity of the core region of the MCS in MONs-HA-Cy5.5 group is as weak as 9.8 (Fig. S11). Whereas the intensity in the core region of the MCS in the SMONs-HA-Cy5.5 group is 17.2, nearly twice the fluorescence signal intensity of its rigid counterpart, implying the SMONs-HA-Cy5.5 can pass through ECM easier and diffuse deeper into the tumor tissues. The fluorescence intensities of all scanning slices were measured to evaluate the penetration performance of SMONs-HA-Cy5.5 and MONs-HA-Cy5.5 in MCSs. As indicated in Fig. 5b, SMONs-HA-Cy5.5 group display more than 2-fold higher fluorescence intensity than MONs-HA-Cy5.5 group throughout the distance of 10 to $90 \mu \mathrm{m}$, suggesting significantly improved tumor penetration ability of SMONs-HA-Cy5.5. In addition, the distributions of the two nanoplatforms in MCSs at the Z-axis distance of $50 \mu \mathrm{m}$ were further analyzed by comparing the gray value of the central linear region (Fig. 5c, horizontal red arrow). For the stiff MONs-HA-Cy5.5, the highest gray value (marked by a vertical blue arrow) appears at $\mathrm{X}$-axis distance of $74 \mu \mathrm{m}$. In comparison, the soft SMONs-HACy5.5 reach their distribution climax (marked by a vertical red arrow) at $104 \mu \mathrm{m}$ distance, clearly demonstrating that a $30 \mu \mathrm{m}$-deeper penetration is achieved in SMONs-HA-Cy5.5 group. Additionally, the grey value of MCS in SMONs-HACy5.5 is always higher than that in MONs-HA-Cy5.5 group from the surface to $300 \mu \mathrm{m}$-distance, which is consistent 
(a)

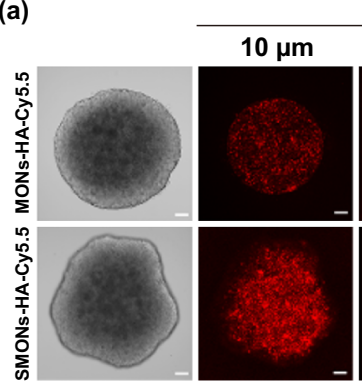

(d)
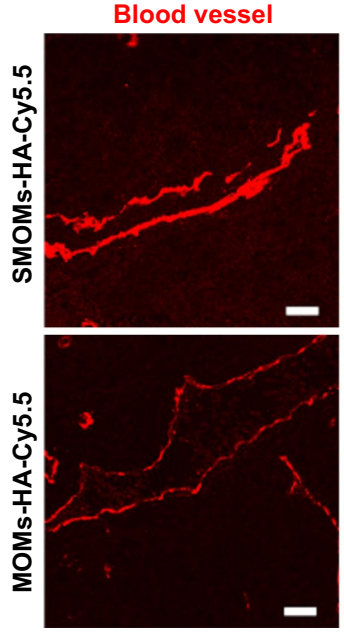

Scanning depth from surface

$30 \mu \mathrm{m}$
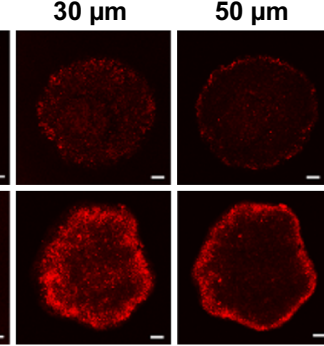

Cy5.5
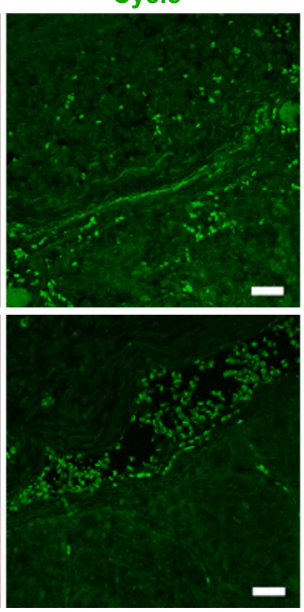

$70 \mu \mathrm{m}$

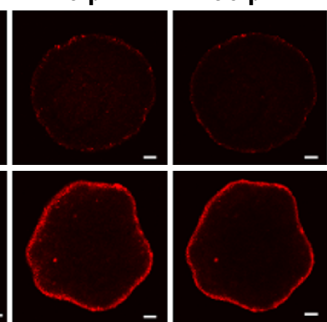

Overlay

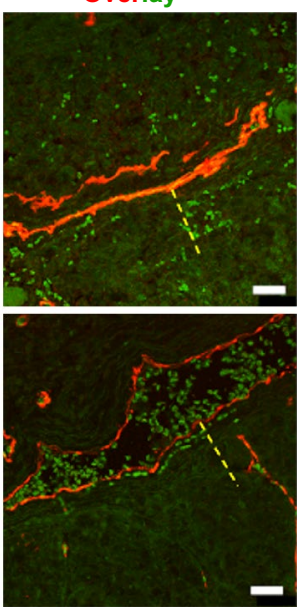

(b)

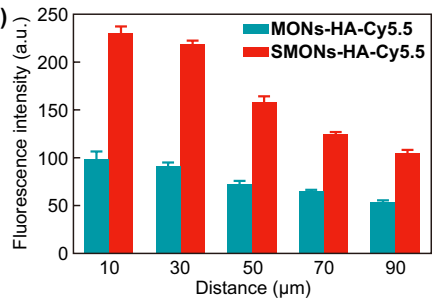

(c)

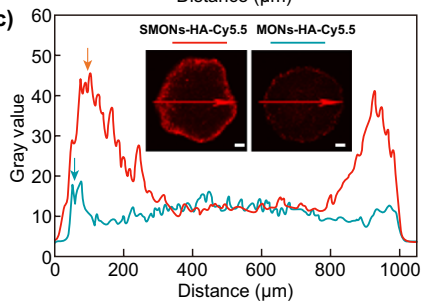

(e)
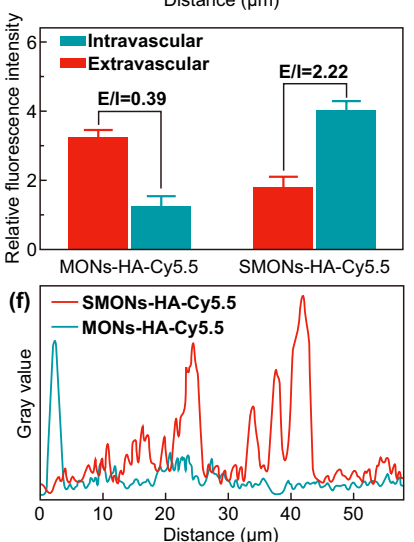

Fig. 5 Penetrating behavior. a CLSM Z-stack scanning images and b corresponding fluorescence intensity of MCF-7 multicellular spheroids (MCSs) upon $4 \mathrm{~h}$ incubation with the MONs-HA-Cy5.5 and SMONs-HA-Cy5.5. Scale bars, $100 \mu \mathrm{m}$. $\mathbf{c}$ Gray value profile of MCSs along the arrow region at the Z-axis distance of $50 \mu \mathrm{m}$. d CLSM images of tumor slices from mice at post- $24 \mathrm{~h}$ injection. Red and green signals were from anti-CD31 stained blood vessels and Cy5.5, respectively. Scale bars, $25 \mu \mathrm{m}$. e Relative Cy5.5 fluorescence intensities of intravascular and extravascular areas. $\mathbf{f}$ The grey value profile of green signal of extravascular areas (indicated by the yellow dashed lines). (Color figure online)

with their higher cellular uptake and deeper penetration. These results clearly demonstrate that the soft SMONsHA-Cy5.5 have tremendous advantage over the stiff ones in tumor penetration.

\subsection{Ex Vivo Intratumoral Distribution}

To compare the ex vivo intratumoral distribution of the two nanoplatforms with different softness, the MCF-7 xenograft tumor-bearing mice were injected with SMONsHA-Cy5.5 or MONs-HA-Cy5.5. After $24 \mathrm{~h}$, the mice were killed and the obtained tumors were sectioned and stained with anti-CD31 antibody to visualize the blood vessels. As can be seen in the CLSM images (Fig. 5d), the green fluorescence signal of Cy5.5 scatters in the tumor interstitium away from the blood vessel in SMONs-HACy5.5 treated group, indicating the soft nanoplatforms can extravasate into perivascular tumor regions and diffuse into deeper tumor tissues. However, for MONs-HA-Cy5.5 treated group, the green fluorescence signal is mainly colocalized with blood vessel or located near the vessel wall, suggesting the poor extravasation and limited penetration. Moreover, the relative Cy5.5 fluorescence intensities (FI) of three intravascular and three extravascular regions were quantitatively analyzed. The extravascular FI/ intravascular FI ratio in SMONs-HA-Cy5.5 treated group is measured to be 2.22, while the value for MONs-HACy5.5 group is only 0.39 (Fig. 5e), demonstrating the soft SMONs-HA-Cy5.5 nanoplatforms have better intratumoral 
extravascular penetration capability. The extravascular distribution of the two nanoplatforms were further evaluated by depicting gray value profile of green signal of an extravascular area (yellow dashed lines). The peak of gray value appears at distance of $2.72 \mu \mathrm{m}$ in MONs-HACy5.5 group (Fig. 5f), implying that the stiff nanoplatforms are confined to the vessel wall. On the contrary, the distribution of the SMONs-HA-Cy5.5 could be observed until $43 \mu \mathrm{m}$ away from the blood vessel, indicating their superior penetration performance. These results demonstrate that soft nanoplatforms have significantly improved extravasation and penetration behavior in tumor tissues. It is speculated that the SMONs-HA-Cy5.5 nanoplatforms can pass through tumor vessel wall fenestrations more easily and diffuse within the tumor ECM more efficiently, which is associated with their soft structure and deformability. In contrast, the MONs-HA-Cy5.5 are constricted within or around the vessels due to their stiffness and nondeformability. The ability of soft nanoplatforms to shrink and deform in dense and confined environments can boost their diffusion compared to non-deformable particles [47].

\subsection{In Vitro PDT of SMONs-HA-Ce6 and MONs-HA-Ce6}

Considering the advantageous biological behavior of the soft SMONs-HA-Cy5.5, we construct a photodynamic nanoplatform by loading a photosensitizer Ce6 in the SMONs-HACy5.5. The loading capacities of the MONs-HA-Cy5.5 and SMONs-HA-Cy5.5 for Ce6 are measured to be $25.7 \mathrm{wt} \%$ and $29.9 \mathrm{wt} \%$, respectively. The hemolytic activities of Ce6 loaded nanoplatforms are lower than $1 \%$ at a concentration of up to $400 \mu \mathrm{g} \mathrm{mL}{ }^{-1}$, indicating excellent hemocompatibility (Fig. S12). The CCK8 cytotoxicity results indicate that the relative viability of MCF-7 cells remains over $80 \%$ after incubation with Ce6 loaded nanoplatforms (Fig. S13a). The ${ }^{1} \mathrm{O}_{2}$ levels in the presence of these nanoplatforms were firstly detected in vitro. After irradiated by a $660 \mathrm{~nm}$ laser for $5 \mathrm{~min}$, the SOSG fluorescence intensities of free Ce6, MONs-HA-Ce6 and SMONs-HA-Ce6 group are measured to be $41,626,34,815,35,918$, respectively (Fig. S13b), suggesting the generation of ${ }^{1} \mathrm{O}_{2}$. Also, the fluorescence intensity elevates gradually along with irradiation time (Fig. $\mathrm{S} 13 \mathrm{c}, \mathrm{d})$. Then the production of ROS was studied at cellular level using DCFH-DA. The fluorescence intensity measured by a microplate reader was the highest when cells were incubated with SMONs-HA-Ce6, which demonstrates the ability of SMONs-HA-Ce6 to generate more ROS in cells (Fig. 6a). Next, a CCK-8 assay was carried out to evaluate their photodynamic effects on MCF-7 cells. After incubation with these nanoplatforms for $24 \mathrm{~h}$ and subsequently received irradiation of a $660 \mathrm{~nm}$ laser for $5 \mathrm{~min}$, the relative cell viabilities show a dose-dependent decrease (Fig. 6b). More notably, the relative cell viabilities of SMONs-HA-Ce6 group are significantly lower than those treated with MONs-HA-Ce6 or free Ce6.

\subsection{In Vivo Antitumor Efficacy}

Then, the in vivo PDT efficacy of the SMONs-HA-Ce6 was evaluated in MCF-7 tumor-bearing mice. As is illustrated in Fig. 6c, the tumor volumes of free $\mathrm{Ce} 6+$ laser group, MONs-HA-Ce6 + laser group and SMONs-HA-Ce6 + laser group are smaller compared to those of PBS, PBS + laser, MONs-HA-Ce6 and SMONs-HA-Ce6 groups, suggesting that PDT can inhibit tumor growth. In detail, the relative tumor volume of SMONs-HA-Ce6 + laser group is 1.46, while the PBS + laser group, free $\mathrm{Ce} 6+$ laser group and MONs-HA-Ce6 + laser group are 10.38, 6.46, and 3.57, respectively. It is obvious that SMONs-HA-Ce6 + laser group reaches an efficacy better than that offered by MONs-HA-Ce6 + laser while free $\mathrm{Ce} 6$ + laser group has the minimal tumor-suppressive efficacy. The significantly improved PDT efficacy of the SMONs-HA-Ce6 is attributed to their higher tumor accumulation and deeper penetration. Mice were killed on day 14, and the excised tumors were weighed. The average tumor weights of the PBS group, MONs-HA-Ce6 group, SMONs-HA-Ce6 group, PBS + laser group, free Ce6 + laser group, MONs-HA$\mathrm{Ce} 6+$ laser group and SMONs-HA-Ce6 + laser group are $1.92,1.90,1.89,1.84,1.26,0.64$, and $0.30 \mathrm{~g}$, respectively (Fig. 6d, e), further confirming that the SMONs-HA-Ce6 group is the most effective in inhibiting tumor growth. The body weights of mice from each group show no significant change, suggesting no major toxicity (Fig. 6f). The antitumor activities were further assessed by analyzing necrosis condition of H\&E staining images of tumor sections at $24 \mathrm{~h}$ post-irradiation. Notably, H\&E images show that enhanced necrosis occurs in tumor tissues of the mice 


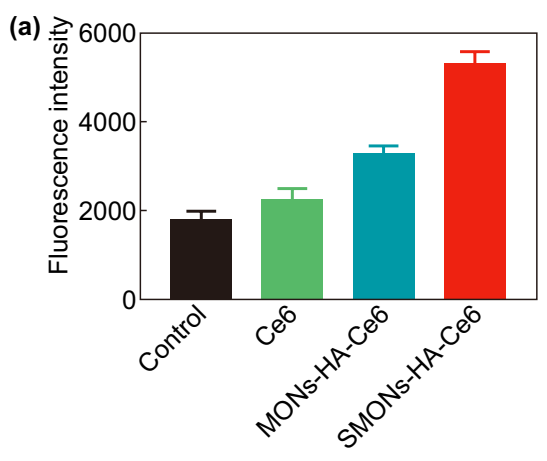

(d)

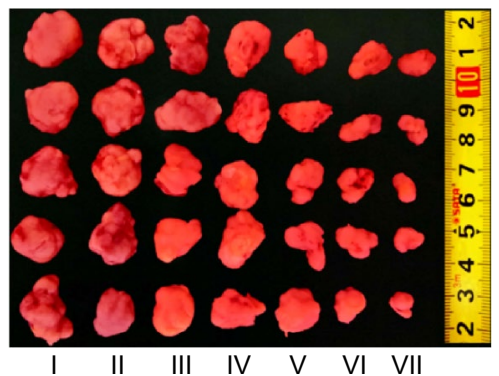

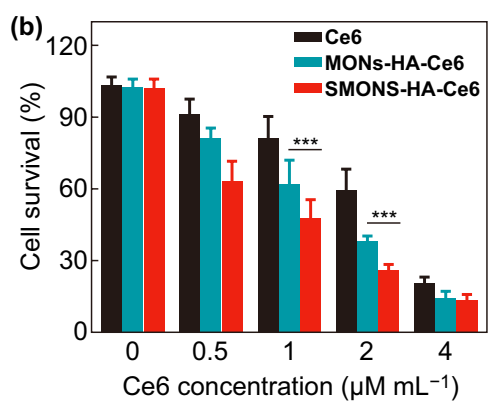

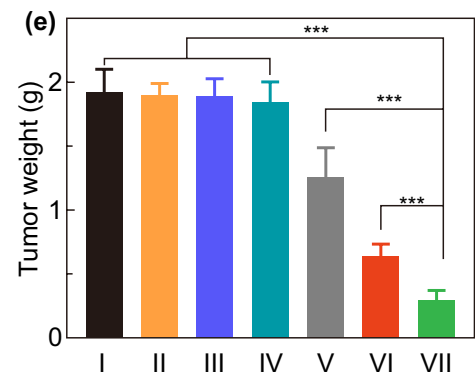

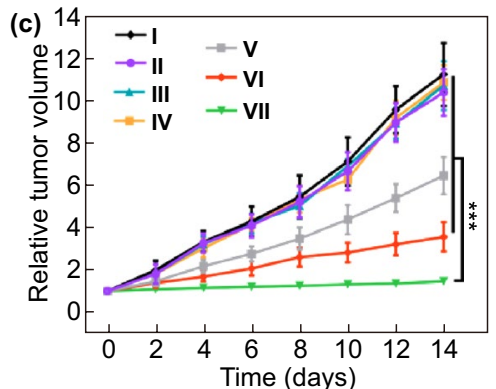

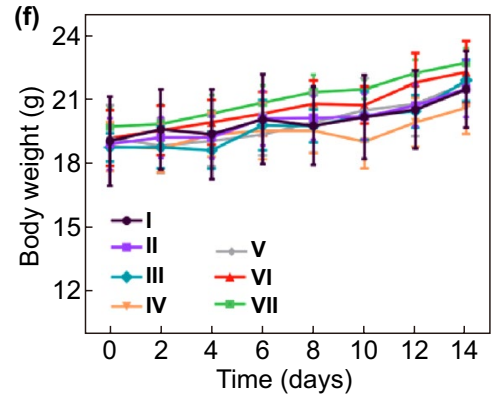

(g)

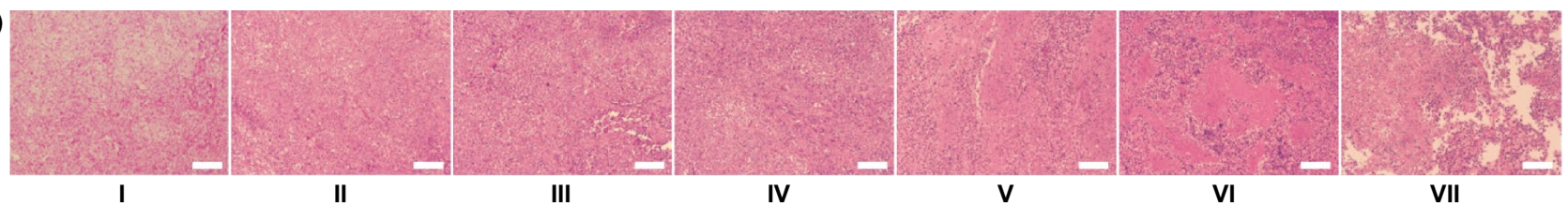

Fig. 6 PDT efficacy. a Fluorescence intensity of DCF in MCF-7 cells, which incubated with different agents and then received laser irradiation (660 $\left.\mathrm{nm}, 0.5 \mathrm{~W} \mathrm{~cm} \mathrm{c}^{-2}, 5 \mathrm{~min}\right)$, measured by a microplate reader. b Viability of MCF-7 cells co-incubated with different concentrations of Ce6, MONs-HA-Ce6, SMONs-HA-Ce6 for $24 \mathrm{~h}$ and then irradiated with laser $\left(660 \mathrm{~nm}, 0.5 \mathrm{~W} \mathrm{~cm}^{-2}\right.$ ) for 5 min. c Tumor growth curves of different groups ( $n=5$ mice per group). d Tumor photographs and e tumor weights at the end point after various treatments. $\mathbf{f}$ The body weights of MCF-7 tumor-bearing mice during treatments. $\mathrm{g} \mathrm{H} \& \mathrm{E}$ staining images of tissue sections from tumors of different groups at $24 \mathrm{~h}$ after irradiation. Scale bars, $100 \mu \mathrm{m}$. $* * * P<0.001$. I: PBS group; II MONs-HA-Ce6 group; III: SMONs-HA-Ce6 group; IV: PBS + laser group; V: free Ce6 + laser group; VI: MONs-HA-Ce6 + laser group; VII: SMONs-HA-Ce6 + laser group

treated with SMONs-HA-Ce6 + laser group compared with MONs-HA-Ce6 + laser group and free Ce6 + laser group, suggesting the improved efficacy of the soft photodynamic nanoplatforms in destroying tumor tissues (Fig. 6g). These results indicate that the soft mesoporous nanoplatform can effectively improve the tumor-suppressive efficiency.

\section{Conclusion}

In summary, we successfully constructed soft mesoporous organosilica nanomaterials harboring a large cavity, cross-wrinkled morphology, and a low Young's modulus
(24.2 MPa). Analysis of their cellular internalization, blood circulation, tumor accumulation/penetration performances reveal that apart from a significantly improved efficiency of cellular uptake in MCF-7 cells, these nanomaterials also exhibit elongated blood circulation, with a fivefold increase in blood drug concentrations compared to stiff ones in the distribution phase, and exhibit drastically higher tumor accumulation. Strikingly, the soft SMONs-HA-Cy5.5 nanomaterials enable higher tumor penetration, reaching $30 \mu \mathrm{m}$-deeper tissue in 3D tumor spheroids. Furthermore, ex vivo intratumoral distribution results indicate that the soft mesoporous nanomaterials significantly improve extravasation behavior and 
intratumoral penetration, generating a 16 -fold increase in diffusion distance in the tumor microenvironment (43 vs. $2.72 \mu \mathrm{m}$ ), relative to stiff counterparts.

Functionally, the soft deformable nanoparticles may have unique fluid mechanics that enable then to reside longer in the circulation system. A relatively longer blood circulation time enhances exposure of soft nanoplatforms to the tumor microvasculature allowing them to accumulate in the tumor site via the EPR effect. During extravasation of nanoparticles, from the systemic circulation to perivascular tumor microenvironment, it is easier for the soft ones to pass through leaky tumor vessels, owing to their deformed nature and improved tumor exposure. During penetration of the tumor parenchyma, soft nanoparticles could deform into ellipsoids, which may contribute to a rotational motion in complex crowded media. This explains why the soft nanoparticles diffuse faster and penetrate deeper into the dense tumor ECM compared to stiff counterparts.

Interestingly, after loading a therapeutic agent $\mathrm{Ce} 6$, the soft mesoporous organosilica nanoplatforms exhibit significantly improved MCF-7 cells killing effect in vitro and bring better therapeutic outcomes for breast cancer in vivo thanks to their higher tumor accumulation and deeper tumor penetration compared to the stiff ones. This is the first study comprehensively exploring the biological performance of soft mesoporous nanoplatforms. Overall, our findings demonstrate tremendous advantages of these nanomaterials, over their stiff counterparts, including improved blood circulation, tumor accumulation/penetration, and photodynamic efficacy. Consequently, the soft mesoporous nanoplatform, reported herein, has the potential for further future development of therapeutic anticancer approaches.

Acknowledgements This work was supported by National Key Basic Research Program of China (973 Program), China (2014CB744504), the National Natural Science Foundation of China, China (81971675, 21603106 and 81971681), and the Natural Science Foundation of Jiangsu Province, China (BK20160017).

Open Access This article is licensed under a Creative Commons Attribution 4.0 International License, which permits use, sharing, adaptation, distribution and reproduction in any medium or format, as long as you give appropriate credit to the original author(s) and the source, provide a link to the Creative Commons licence, and indicate if changes were made. The images or other third party material in this article are included in the article's Creative Commons licence, unless indicated otherwise in a credit line to the material. If material is not included in the article's Creative Commons licence and your intended use is not permitted by statutory regulation or exceeds the permitted use, you will need to obtain permission directly from the copyright holder. To view a copy of this licence, visit http://creativecommons.org/licenses/by/4.0/.

Electronic supplementary material The online version of this article (https://doi.org/10.1007/s40820-020-00465-7) contains supplementary material, which is available to authorized users.

\section{References}

1. S. Wang, X. Ma, X. Hong, Y. Cheng, Y. Tian et al., Adjuvant photothermal therapy inhibits local recurrences after breastconserving surgery with little skin damage. ACS Nano 12(1), 662-670 (2018). https://doi.org/10.1021/acsnano.7b07757

2. Y. Yang, J. Tang, M. Zhang, Z. Gu, H. Song, Y. Yang, C. Yu, Responsively aggregatable sub-6 nm nanochelators induce simultaneous antiangiogenesis and vascular obstruction for enhanced tumor vasculature targeted therapy. Nano Lett. 19(11), 7750-7759 (2019). https://doi.org/10.1021/acs.nanol ett.9b02691

3. D. Li, S. He, Y. Wu, J. Liu, Q. Liu et al., Excretable lanthanide nanoparticle for biomedical imaging and surgical navigation in the second near-infrared window. Adv. Sci. 6(23), 1902042 (2019). https://doi.org/10.1002/advs.201902042

4. H. Chen, J. Wang, X. Feng, M. Zhu, S. Hoffmann et al., Mitochondria-targeting fluorescent molecules for high efficiency cancer growth inhibition and imaging. Chem. Sci. 10(34), 7946-7951 (2019). https://doi.org/10.1039/c9sc01410a

5. S.K. Golombek, J.N. May, B. Theek, L. Appold, N. Drude, F. Kiessling, T. Lammers, Tumor targeting via EPR: strategies to enhance patient responses. Adv. Drug Deliv. Rev. 130, 17-38 (2018). https://doi.org/10.1016/j.addr.2018.07.007

6. H.F. Wang, R. Ran, Y. Liu, Y. Hui, B. Zeng et al., Tumor-vasculature-on-a-chip for investigating nanoparticle extravasation and tumor accumulation. ACS Nano 12(11), 11600-11609 (2018). https://doi.org/10.1021/acsnano.8b06846

7. J. Liu, P. Du, H. Mao, L. Zhang, H. Ju, J. Lei, Dual-triggered oxygen self-supply black phosphorus nanosystem for enhanced photodynamic therapy. Biomaterials 172, 83-91 (2018). https ://doi.org/10.1016/j.biomaterials.2018.04.051

8. M. Satpathy, L. Wang, R.J. Zielinski, W. Qian, Y.A. Wang et al., Targeted drug delivery and image-guided therapy of heterogeneous ovarian cancer using her2-targeted theranostic nanoparticles. Theranostics 9(3), 778-795 (2019). https://doi. org/10.7150/thno.29964

9. D. Huo, X. Jiang, Y. Hu, Recent advances in nanostrategies capable of overcoming biological barriers for tumor management. Adv. Mater. (2019). https://doi.org/10.1002/adma.20190 4337

10. J. Shi, P.W. Kantoff, R. Wooster, O.C. Farokhzad, Cancer nanomedicine: progress, challenges and opportunities. Nat. Rev. Cancer 17(1), 20-37 (2017). https://doi.org/10.1038/ nrc.2016.108 
11. Y.S. Youn, Y.H. Bae, Perspectives on the past, present, and future of cancer nanomedicine. Adv. Drug Deliv. Rev. 130, 3-11 (2018). https://doi.org/10.1016/j.addr.2018.05.008

12. H.H. Gustafson, D. Holt-Casper, D.W. Grainger, H. Ghandehari, Nanoparticle uptake: the phagocyte problem. Nano Today 10(4), 487-510 (2015). https://doi.org/10.1016/j.nanto d.2015.06.006

13. Q. Dai, S. Wilhelm, D. Ding, A.M. Syed, S. Sindhwani et al., Quantifying the ligand-coated nanoparticle delivery to cancer cells in solid tumors. ACS Nano 12(8), 8423-8435 (2018). https://doi.org/10.1021/acsnano.8b03900

14. M.W. Dewhirst, T.W. Secomb, Transport of drugs from blood vessels to tumour tissue. Nat. Rev. Cancer 17(12), 738-750 (2017). https://doi.org/10.1038/nrc.2017.93

15. H. Zhou, Z. Fan, P.Y. Li, J. Deng, D.C. Arhontoulis et al., Dense and dynamic polyethylene glycol shells cloak nanoparticles from uptake by liver endothelial cells for long blood circulation. ACS Nano 12(10), 10130-10141 (2018). https:// doi.org/10.1021/acsnano.8b04947

16. P. Jin, R. Sha, Y. Zhang, L. Liu, Y. Bian et al., Blood circulation-prolonging peptides for engineered nanoparticles identified via phage display. Nano Lett. 19(3), 1467-1478 (2019). https://doi.org/10.1021/acs.nanolett.8b04007

17. M. Huo, W. Li, A.S. Chaudhuri, Y. Fan, X. Han, C. Yang, Z. Wu, X. Qi, Bio-stimuli-responsive multi-scale hyaluronic acid nanoparticles for deepened tumor penetration and enhanced therapy. Carbohydr. Polym. 171, 173-182 (2017). https://doi. org/10.1016/j.carbpol.2017.05.017

18. G. Yang, S.Z.F. Phua, W.Q. Lim, R. Zhang, L. Feng et al., A hypoxia-responsive albumin-based nanosystem for deep tumor penetration and excellent therapeutic efficacy. Adv. Mater. 31(25), 1901513 (2019). https://doi.org/10.1002/adma.20190 1513

19. J. Kim, C. Jo, W.G. Lim, S. Jung, Y.M. Lee et al., Programmed nanoparticle-loaded nanoparticles for deep-penetrating 3d cancer therapy. Adv. Mater. 30(29), 1707557 (2018). https://doi.org/10.1002/adma.201707557

20. S. Wang, Y. Tian, W. Tian, J. Sun, S. Zhao et al., Selectively sensitizing malignant cells to photothermal therapy using a cd44-targeting heat shock protein 72 depletion nanosystem. ACS Nano 10(9), 8578-8590 (2016). https://doi. org/10.1021/acsnano.6b03874

21. J. Mougin, S.O. Yesylevskyy, C. Bourgaux, D. Chapron, J.P. Michel et al., Stacking as a key property for creating nanoparticles with tunable shape: the case of squalenoyldoxorubicin. ACS Nano 13(11), 12870-12879 (2019). https ://doi.org/10.1021/acsnano.9b05303

22. D. Wang, C. Gao, W. Wang, M. Sun, B. Guo, H. Xie, Q. He, Shape-transformable, fusible rodlike swimming liquid metal nanomachine. ACS Nano 12(10), 10212-10220 (2018). https ://doi.org/10.1021/acsnano.8b05203

23. L. Yang, Z. Zhou, J. Song, X. Chen, Anisotropic nanomaterials for shape-dependent physicochemical and biomedical applications. Chem. Soc. Rev. 48(19), 5140-5176 (2019). https://doi.org/10.1039/c9cs00011a
24. Z. Zhang, C. Liu, C. Li, W. Wu, X. Jiang, Shape effects of cylindrical versus spherical unimolecular polymer nanomaterials on in vitro and in vivo behaviors. Research (Washington, DC) 2019, 2391486 (2019). https://doi.org/10.34133 /2019/2391486

25. H.J. Li, J.Z. Du, J. Liu, X.J. Du, S. Shen et al., Smart superstructures with ultrahigh $\mathrm{pH}$-sensitivity for targeting acidic tumor microenvironment: instantaneous size switching and improved tumor penetration. ACS Nano 10(7), 6753-6761 (2016). https://doi.org/10.1021/acsnano.6b02326

26. J. Wang, W. Mao, L.L. Lock, J. Tang, M. Sui et al., The role of micelle size in tumor accumulation, penetration, and treatment. ACS Nano 9(7), 7195-7206 (2015). https://doi. org/10.1021/acsnano.5b02017

27. T. Zhao, P. Wang, Q. Li, A.A. Al-Khalaf, W.N. Hozzein, F. Zhang, X. Li, D. Zhao, Near-infrared triggered decomposition of nanocapsules with high tumor accumulation and stimuli responsive fast elimination. Angew. Chem. Int. Ed. 57(10), 2611-2615 (2018). https://doi.org/10.1002/ anie. 201711354

28. J.S. Suk, Q. Xu, N. Kim, J. Hanes, L.M. Ensign, Pegylation as a strategy for improving nanoparticle-based drug and gene delivery. Adv. Drug Deliv. Rev. 99(Pt A), 28-51 (2016). https ://doi.org/10.1016/j.addr.2015.09.012

29. K.L. Zhang, J. Zhou, H. Zhou, Y. Wu, R. Liu et al., Bioinspired "active" stealth magneto-nanomicelles for theranostics combining efficient MRI and enhanced drug delivery. ACS Appl. Mater. Interfaces. 9(36), 30502-30509 (2017). https:// doi.org/10.1021/acsami.7b10086

30. Y. Zou, Y. Liu, Z. Yang, D. Zhang, Y. Lu et al., Effective and targeted human orthotopic glioblastoma xenograft therapy via a multifunctional biomimetic nanomedicine. Adv. Mater. 30(51), 1803717 (2018). https://doi.org/10.1002/adma.20180 3717

31. Y. Chen, J. Shi, Chemistry of mesoporous organosilica in nanotechnology: molecularly organic-inorganic hybridization into frameworks. Adv. Mater. 28(17), 3235-3272 (2016). https ://doi.org/10.1002/adma.201505147

32. T.L. Nguyen, Y. Choi, J. Kim, Mesoporous silica as a versatile platform for cancer immunotherapy. Adv. Mater. 31(34), 1803953 (2019). https://doi.org/10.1002/adma.201803953

33. Z. Teng, W. Li, Y. Tang, A. Elzatahry, G. Lu, D. Zhao, Mesoporous organosilica hollow nanoparticles: synthesis and applications. Adv. Mater. 31(38), 1707612 (2019). https://doi. org/10.1002/adma.201707612

34. L. Li, Y. Lu, C. Jiang, Y. Zhu, X. Yang et al., Actively targeted deep tissue imaging and photothermal-chemo therapy of breast cancer by antibody-functionalized drug-loaded x-ray-responsive bismuth sulfide@mesoporous silica core-shell nanoparticles. Adv. Funct. Mater. 28(5), 1704623 (2018). https://doi. org/10.1002/adfm.201704623

35. M. Huo, L. Wang, Y. Chen, J. Shi, Tumor-selective catalytic nanomedicine by nanocatalyst delivery. Nat. Commun. 8(1), 357 (2017). https://doi.org/10.1038/s41467-017-00424-8

36. Y. Lu, Y. Yang, Z. Gu, J. Zhang, H. Song, G. Xiang, C. Yu, Glutathione-depletion mesoporous organosilica nanoparticles 
as a self-adjuvant and co-delivery platform for enhanced cancer immunotherapy. Biomaterials 175, 82-92 (2018). https:// doi.org/10.1016/j.biomaterials.2018.05.025

37. Z. Teng, C. Wang, Y. Tang, W. Li, L. Bao et al., Deformable hollow periodic mesoporous organosilica nanocapsules for significantly improved cellular uptake. J. Am. Chem. Soc. 140(4), 1385-1393 (2018). https://doi.org/10.1021/jacs.7b106 94

38. Q. Chen, L. Feng, J. Liu, W. Zhu, Z. Dong, Y. Wu, Z. Liu, Intelligent albumin-mno2 nanoparticles as $\mathrm{PH}-/ \mathrm{H} 2 \mathrm{O} 2$-responsive dissociable nanocarriers to modulate tumor hypoxia for effective combination therapy. Adv. Mater. 28(33), 7129-7136 (2016). https://doi.org/10.1002/adma.201601902

39. Z. Teng, X. Su, B. Lee, C. Huang, Y. Liu et al., Yolk-shell structured mesoporous nanoparticles with thioether-bridged organosilica frameworks. Chem. Mater. 26(20), 5980-5987 (2014). https://doi.org/10.1021/cm502777e

40. B.P. Jiang, L. Zhang, X.L. Guo, X.C. Shen, Y. Wang, Y. Zhu, H. Liang, Poly(n-phenylglycine)-based nanoparticles as highly effective and targeted near-infrared photothermal therapy/photodynamic therapeutic agents for malignant melanoma. Small 13(8), 1602496 (2017). https://doi.org/10.1002/smll.20160 2496

41. Q. Sun, Z. Zhou, N. Qiu, Y. Shen, Rational design of cancer nanomedicine: nanoproperty integration and synchronization. Adv. Mater. 29(14), 1606628 (2017). https://doi.org/10.1002/ adma.201606628
42. Y. Hui, X. Yi, F. Hou, D. Wibowo, F. Zhang, D. Zhao, H. Gao, C.X. Zhao, Role of nanoparticle mechanical properties in cancer drug delivery. ACS Nano 13(7), 7410-7424 (2019). https://doi.org/10.1021/acsnano.9b03924

43. A.C. Anselmo, S. Mitragotri, Impact of particle elasticity on particle-based drug delivery systems. Adv. Drug Deliv. Rev. 108, 51-67 (2017). https://doi.org/10.1016/j.addr.2016.01.007

44. K.M. Tsoi, S.A. MacParland, X.Z. Ma, V.N. Spetzler, J. Echeverri et al., Mechanism of hard-nanomaterial clearance by the liver. Nat. Mater. 15(11), 1212-1221 (2016). https://doi. org/10.1038/nmat4718

45. S. Wilhelm, A.J. Tavares, Q. Dai, S. Ohta, J. Audet, H.F. Dvorak, W.C.W. Chan, Analysis of nanoparticle delivery to tumours. Nat. Rev. Mater. (2016). https://doi.org/10.1038/natre vmats. 2016.14

46. C. Xu, Z. Teng, Y. Zhang, L. Yuwen, Q. Zhang et al., Flexible $\mathrm{MoS}_{2}$-embedded human serum albumin hollow nanocapsules with long circulation times and high targeting ability for efficient tumor ablation. Adv. Funct. Mater. 28(45), 1804081 (2018). https://doi.org/10.1002/adfm.201804081

47. P.L. Latreille, V. Adibnia, A. Nour, J.M. Rabanel, A. Lalloz et al., Spontaneous shrinking of soft nanoparticles boosts their diffusion in confined media. Nat. Commun. 10(1), 4294 (2019). https://doi.org/10.1038/s41467-019-12246-X 